TRANSACTIONS OF THE

AMERICAN MATHEMATICAL SOCIETY

Volume 365, Number 8, August 2013, Pages 4153-4179

S 0002-9947(2013)05764-9

Article electronically published on February 14, 2013

\title{
FRAMED MODULI AND GRASSMANNIANS OF SUBMODULES
}

\author{
STANISLAV FEDOTOV
}

\begin{abstract}
In this work we study a realization of moduli spaces of framed quiver representations as Grassmannians of submodules devised by Markus Reineke. Obtained is a generalization of this construction to finite dimensional associative algebras and for quivers with oriented cycles over an arbitrary infinite field. As an application we get an explicit realization of fibers for the moduli space bundle over the categorical quotient for the quiver $A_{n-1}^{(1)}$ and the ground fields $\mathbb{C}$ and $\mathbb{R}$.
\end{abstract}

\section{INTRODUCTION}

A quiver $Q$ is a diagram of arrows determined by two finite sets $Q_{0}$ (the set of "vertices") and $Q_{1}$ (the set of "arrows") with two maps $h, t: Q_{1} \rightarrow Q_{0}$ which indicate the vertices at the head and tail of each arrow. A representation $(W, \varphi)$ of $Q$ consists of a collection of finite dimensional $\mathbb{k}$-vector spaces $W_{i}$, for each $i \in Q_{0}$, together with linear maps $\varphi_{a}: W_{t a} \rightarrow W_{h a}$, for each $a \in Q_{1}$. The dimension vector $\alpha \in \mathbb{Z}^{Q_{0}}$ of such a representation is given by $\alpha_{i}=\operatorname{dim}_{\mathbb{k}} W_{i}$. A morphism $f:\left(W_{i}, \varphi_{a}\right) \rightarrow\left(U_{i}, \psi_{a}\right)$ of representations consists of linear maps $f_{i}: W_{i} \rightarrow U_{i}$, for each $i \in Q_{0}$ such that $f_{h a} \varphi_{a}=\psi_{a} f_{t a}$, for each $a \in Q_{1}$. Evidently, it is an isomorphism if and only if each $f_{i}$ is as well. Thus, isomorphism classes of representations of $Q$ with dimension vector $\alpha$ coincide with orbits of the action of $G L(\alpha)=\prod_{i \in Q_{0}} G L_{i}(\mathbb{k})$ on the representation space $\operatorname{Rep}(Q, \alpha)$.

In studying quiver representations standard approaches of invariant theory often fail because the algebra of invariants is poor or even trivial, as in the case of the quiver without oriented cycles, and so the categorical quotient $\operatorname{Rep}(Q, \alpha) / / G L(\alpha):=$ $\operatorname{Spec} \mathbb{k}[\operatorname{Rep}(Q, \alpha)]^{G L(\alpha)}$ is a point. Constructions of geometric invariant theory may help to compensate this defect. Indeed, one can consider the trivial linearization twisted by a character $\chi$ of $G L(\alpha)$, which restricts our attention to an open subset of $\operatorname{Rep}(Q, \alpha)$, consisting of $\chi$-semistable representations. Within the open set there are more closed orbits, and the corresponding algebraic quotient is more interesting. In his paper 9] A. D. King showed that the notions of semistability and stability, that arise from geometric invariant theory, coincide with more algebraic notions, expressed in the language of abelian categories. Namely, he devised a link between this concept and the so-called $\theta$-stability. All the characters of $G L(\alpha)$ are given by $\chi_{\theta}(g)=\prod_{i \in Q_{0}} \operatorname{det}\left(g_{i}\right)^{\theta_{i}}$ for $\theta \in \mathbb{Z}^{Q_{0}}$, and a representation $W$ is a $\chi_{\theta}$-(semi)stable point of the space $\operatorname{Rep}(Q, \alpha)$ if and only if it is $\theta$-(semi)stable as an element of the abelian category $\operatorname{Rep}(Q)$ (see [9, Section 2]). King proved the existence of

Received by the editors December 28, 2010 and, in revised form, November 11, 2011. 2010 Mathematics Subject Classification. Primary 14D22; Secondary 16G10, 16G20.

This work was supported by grant RFFI 09-01-90416 - Ukr-f-a. 
coarse moduli space for semistable representations of quivers and guaranteed that for stable representations there is a fine moduli space. This technique allowed a straightforward and convenient adaptation to the study of representations of finite dimensional algebras [9, Section 4]. The approach used by King was generalized and reformulated in a more flexible form by A. N. Rudakov [14, §3].

An alternative approach to the problem considered was introduced by B. HuisgenZimmermann [7. Let $A$ be a finite dimensional associative algebra with unity. Fix a semisimple $A$-module $T$ with a projective cover $P$ and a positive integer $m$. Denote by $\mathfrak{G}_{\mathfrak{a s s}} \mathfrak{s}_{m}^{T}$ the Grassmannian of all $(\operatorname{dim} P-m)$-dimensional submodules of the radical $\operatorname{rad} P$. There is then a bijection between Aut $_{A} P$-orbits in $\mathfrak{G r a s s}_{m}^{T}$ and isomorphism classes of $m$-dimensional $A$-modules with top isomorphic to $T$, sending $\operatorname{Aut}_{A} P \cdot C$ to the isomorphism class of $P / C$. Investigated in [7] are such triples $(A, T, m)$, where $\mathfrak{G} \mathfrak{r a s s}_{m}^{T}$ itself provides a moduli space classifying the $d$ dimensional $A$-modules with top $T$, up to isomorphism. It is also proved there that $\mathfrak{G r a s s}_{m}^{T}$ admits an open covering by representation-theoretically defined affine charts. For further generalization and a systematical treatment of the whole hierarchy of moduli-parametrizing Grassmannian varieties, see 8 .

Another possible way of applying invariant theory to the study of quiver representations is to consider framed representations, thus achieving better precision at the expense of extending the representation space. They first appeared in [12] as one of the steps in the construction of Nakajima varieties.

Let $Q$ be a quiver and $\alpha$ be a dimension vector. Fix an additional dimension vector $\zeta$ and consider the space $\operatorname{Rep}(Q, \alpha, \zeta):=\operatorname{Rep}(Q, \alpha) \oplus \bigoplus_{i \in Q_{0}} \operatorname{Hom}_{\mathbb{k}}\left(\mathbb{k}^{\alpha_{i}}, \mathbb{k}^{\zeta_{i}}\right)$. Its elements are said to be framed representations of $Q$. Define a $G L(\alpha)$-action on $\operatorname{Rep}(Q, \alpha, \zeta)$ by $g \cdot\left(M,\left(f_{i}\right)_{i=1}^{n}\right)=\left(g \cdot M,\left(f_{i} g_{i}^{-1}\right)_{i=1}^{n}\right)$. A framed representation $(M, f)$ is called stable if there is no nonzero subrepresentation $N$ of $M$ which is contained in ker $f$. Denote by $\operatorname{Rep}^{s}(Q, \alpha, \zeta)$ the space of stable framed representations. One can show that the quotient of $\operatorname{Rep}^{s}(Q, \alpha, \zeta)$ is more efficient in discriminating orbits than the standard categorical quotient. Furthermore, it can be shown that it is in a sense reducible to King's construction and thus enjoys all its properties.

Grassmanians of submodules of injective modules arise independently in the course of this approach. In [13] M. Reineke obtained for acyclic quivers a realization of framed moduli space as a Grassmannian of subrepresentations of an injective representation depending only on dimension vectors $\alpha$ and $\zeta$. He further investigated its cohomology (see also [3]) and applications to quantum groups.

The aim of this work is to explore possible generalizations of Reineke's construction. So far, there are two of them. First, we can adapt the construction for quivers with relations, i.e. at least for finite dimensional algebras. We may also try to eliminate the condition of having no oriented cycles. Both possibilities are discussed below.

Section 2 is devoted to giving some basic results on the connection between finite dimensional algebras and quivers. In Subsection 3.1 we introduce the notion of a framed representation space for finite dimensional algebras.

A straightforward generalization of Reineke's ideas to finite dimensional algebras is presented in Subsection 3.2. We prove that for a finite dimensional algebra $A$ and two dimension vectors $\alpha$ and $\zeta$ the quotient space $\operatorname{Rep}^{s}(A, \alpha, \zeta) / / G L(\alpha)$ is isomorphic to the Grassmannian of submodules of a certain injective $A$-module $J$. 
In Sections 4 to 6 we investigate a possible way to generalize this result to quivers with oriented cycles. It is crucial for Reineke's construction that $A$ is finite dimensional, since otherwise we are no longer able to embed all stable framed representations in a finite dimensional module. In general, we thus come to consider Grassmannians of submodules in infinite dimensional spaces, which makes it impossible to apply the usual techniques. So, having established a bijection between such a Grassmannian and the moduli space we provide the former with a structure of algebraic variety, but this gives us no information about the moduli space itself. Hence we are forced to restrict our attention to fibers of the moduli space bundle over the categorical quotient.

In their work [6] J. Engel and M. Reineke, using Luna's stratification, described fibers of the bundle for $\mathbb{k}=\mathbb{C}$ and for arbitrary quivers as nilpotent parts of the framed moduli space for some new quiver $Q^{\prime}$ and dimension vectors $\alpha^{\prime}$ and $\zeta^{\prime}$ (see [6. Theorem 4.1]). Moreover, it follows from their results that fibers over points of a Luna stratum are isomorphic to each other. Theorem 3.5 together with Proposition 3.10 thus imply that each fiber of $\pi_{s}$ is isomorphic to the Grassmannian of submodules of a module over some finite dimensional algebra with a certain dimension vector.

We try to make our approach work over arbitrary infinite fields. Without the assumption on the ground field the results of Engel and Reineke are not true in the way in which they are stated. At least for $\mathbb{k}=\mathbb{R}$ we give such an example in Section 5. Moreover, one should be careful when trying to apply constructions of invariant theory. For this reason we prefer to say that we construct a moduli space of framed representations with given values of basic invariant functions.

In Sections 4 and 5 we study the quiver $A_{n-1}^{(1)}$ with cyclic orientation. We obtain an embedding of $\operatorname{Rep}^{s}\left(A_{n-1}^{(1)}, \alpha, \zeta\right)$ into an infinite dimensional representation $J$. Recall that by Procesi-Razmyslov's Theorem [11, Theorem 1] (see also [4] and [15] for generalizations to arbitrary infinite fields) the algebra of invariant polynomial functions on $\operatorname{Rep}(Q, \alpha, \zeta)$ is generated by traces of oriented cycles in $Q$. Since in $Q=A_{n-1}^{(1)}$ there is only one cycle $\tau_{i}$ of minimal length starting at each vertex $i \in Q_{0}$, traces of $\tau_{i}^{k}$ are polynomials in coefficients of characteristic polynomials $\chi_{i}$ of $\tau_{i}$. So, we consider a map $\pi: \operatorname{Rep}^{s}(Q, \alpha, \zeta) \rightarrow(\mathbb{C}[t])^{n}$, taking a pair $(M, f)$ to a tuple of characteristic polynomials of $\tau_{i}$, and investigate its fibers. Let $\bar{\chi}=$ $\left(\chi_{1}, \ldots, \chi_{n}\right)$, and also let $h_{0}(x)=x, h_{1}(x), \ldots, h_{N}(x)$ be all different irreducible factors of $\chi_{1}, \ldots, \chi_{n}$ and $r_{i j}$ be the multiplicity of $h_{j}$ as a factor of $h_{i}$ (we allow that $\left.r_{i 0}=0\right)$. For each $j=0,1, \ldots, N$ consider a new dimension vector $\underline{r}_{j}$ with $\left(\underline{r}_{j}\right)_{i}=r_{i j}$. It turns out that images of representations from $\pi^{-1}(\bar{\chi})$ lie in the direct sum of finite dimensional submodules $J\left(h_{j}, \underline{r}_{j}\right) \subseteq J$. Moreover, it may be shown that the moduli space $\pi^{-1}(\bar{\chi}) / / G L(\alpha)$ is isomorphic to the product of the Grassmannians of their subrepresentations. The details are given in Section 4. In Section 5 we obtain an explicit presentation by equations in projective space of fibers of the moduli space bundle over the categorical quotient for ground field $\mathbb{C}$ or $\mathbb{R}$. Section 6 is devoted to applying this technique to any quiver $Q$ where all oriented cycles pairwise commute. The main result here is that for every $y \in$ $\operatorname{Spec} \mathbb{k}[\operatorname{Rep}(Q, \alpha, \zeta)]^{G L(\alpha)}$ and two dimension vectors $\alpha$ and $\zeta$ there is a quiver $Q^{\boldsymbol{N}}$, a dimension vector $\widetilde{\alpha} \in\left(\mathbb{Z}_{\geqslant 0}\right)^{Q_{0}^{\star}}$, and a finite dimensional representation $W^{\wedge}$ of 
$Q^{\text {s }}$ such that the moduli space of representations belonging to the fiber $\pi^{-1}(y)$ is isomorphic to the Grassmannian of $\widetilde{\alpha}$-dimensional submodules of $W$

The author thanks his supervisor, Ivan Arzhantsev, for useful discussions. He also thanks Markus Reineke, who brought to his attention the paper [6] and the universal description of fibers of $\pi_{s}$. He would also like to thank the referee for a careful reading of the manuscript and many valuable remarks.

\section{BACKGROUND INFORMATION}

First, recall some general facts concerning finite dimensional algebras and their connection with quivers.

Let $A$ be an associative finite dimensional algebra over an arbitrary field $\mathbb{k}$. An element $e \in A$ is called idempotent if $e^{2}=e$. We say that two idempotents $e_{1}$ and $e_{2}$ are orthogonal if $e_{1} e_{2}=e_{2} e_{1}=0$. An idempotent $e$ is primitive if it is not a sum of two nonzero orthogonal idempotents. It is well known that for an algebra $A$ with unity there always exists a decomposition $1=e_{1}+\ldots+e_{n}$, where $e_{i}$ are primitive orthogonal idempotents. Note that this decomposition induces a decomposition $A=A e_{1} \oplus \ldots \oplus A e_{n}$ of the regular $A$-module called Peirce decomposition.

An algebra is said to be splitting if the quotient $A / \mathfrak{r}$, where $\mathfrak{r}$ stands for the radical of $A$, is isomorphic to a direct product of matrix algebras over the ground field. Note that all algebras over an algebraically closed field are splitting. An algebra is called basic if $A / \mathfrak{r}$ is isomorphic to a direct product of division rings. This condition is equivalent to the following: in the decomposition $A=P_{1} \oplus \ldots \oplus P_{k}$ of the regular module, where all $P_{i}$ are indecomposable projective modules, all the summands are pairwise nonisomorphic [5, Theorem 3.5.4].

Fix a decomposition $1=e_{1}+\ldots+e_{n}$ of the unity $1 \in A$, where all $e_{i}$ are primitive orthogonal idempotents. It is not hard to see that every $A$-module $M$ as a $\mathbb{k}$-vector space may be decomposed as $M=\bigoplus_{i=1}^{n} M_{i}$, where $M_{i}=e_{i} M$. The dimension vector of $M$ is the vector $\alpha=\operatorname{dim} M$ with $\alpha_{i}=\operatorname{dim} M_{i}$. Decomposing the ideals $A e_{i}$ of $A$ (which are submodules of the regular module) in this way, we obtain the two-sided Peirce decomposition of $A: A=\bigoplus_{i, j} e_{j} A e_{i}$. The components $e_{j} A e_{i}$ are neither left nor right ideals, but they provide a convenient matrix interpretation of the elements of $A$ (see [5, Chapter $1, \S 7]$ ).

The set of all $A$-modules with dimension vector $\alpha$ will be denoted by $\operatorname{Rep}(A, \alpha)$. The group $G L(\alpha)=\prod_{i=1}^{n} G L_{\alpha_{i}}(\mathbb{k})$ acts on this set, each factor acting by base change in $M_{i}$. Namely, for an element $a \in e_{j} A e_{i}$ the corresponding operator $\varphi(a)$ of the representation $A \rightarrow L(M)$ maps $M_{i}$ to $M_{j}$ and all the rest of the components to zero. Thus we may define the action $g=\left(g_{t}\right)_{t=1}^{n} \in \prod_{i=1}^{n} G L\left(M_{i}\right)=G L(\alpha)$ as follows: $(g \cdot \varphi(a))(m)=\left(g_{j} \varphi(a) g_{i}^{-1}\right)(m)$, for all $g \in G, m \in M_{i}$. Since $A$ admits the two-sided Peirce decomposition $A=\bigoplus_{i, j} e_{j} A e_{i}$, the action is well defined.

Now we recall the connection between algebras and quivers. Let $\mathbb{k}$ be a field. For a quiver $Q$ one defines a path algebra $\mathbb{k} Q$. As a linear space it is the span of all paths in $Q$, including those of length 0 , which we identify with vertices of $Q$. Multiplication in $\mathbb{k} Q$ is defined by

$$
\sigma \cdot \tau=\left\{\begin{array}{l}
\sigma \tau, \text { if it is a path in } Q, \\
0, \text { otherwise, }
\end{array}\right.
$$

for two paths $\sigma$ and $\tau$ in $Q$. 
A relation in $Q$ is a $\mathbb{k}$-linear combination of paths in $Q$ of length not less than 2 with the same source and target. For a set of relations $\rho$ denote by $\langle\rho\rangle$ the ideal of the algebra $\mathbb{k} Q$ generated by these relations.

Proposition 2.1 ([1, Proposition II.2.5]). For a finite dimensional algebra $A$ the category of finitely generated A-modules is equivalent to the category of finitely generated $\Gamma$-modules for some basic algebra $\Gamma$.

Thus the problem of classifying the representations of arbitrary finite dimensional algebras can be in a sense reduced to the case of basic algebras. So we will be considering only basic algebras.

Let us briefly recall the procedure of assigning a quiver $Q(A)$ to a splitting basic algebra $A$. Let $\mathfrak{r}$ be the radical of $A, 1=\bar{e}_{1}+\ldots+\bar{e}_{n}$ be a decomposition of unity in $A / \mathfrak{r}$, and $1=e_{1}+\ldots+e_{n}$ the corresponding decomposition of unity in $A$; further, let $W=\left(\mathfrak{r} / \mathfrak{r}^{2}\right)$. Denote $t_{i j}=\operatorname{dim}_{\mathbb{k}} e_{j} W e_{i}$. Now set $Q(A)=\left(Q_{0}, Q_{1}\right)$ with $Q_{0}=\{1, \ldots, n\}$ and $t_{i j}$ arrows from the $i$-th vertex to the $j$-th one.

Let $(\mathbb{k} Q)_{\geqslant 1}$ be the ideal generated by the arrows in $Q$. An ideal $I \triangleleft \mathbb{k} Q$ is said to be regular if $(\mathbb{k} Q)_{\geqslant 1}^{2} \supseteq I \supseteq(\mathbb{k} Q)_{\geqslant 1}^{t}$, for some $t \geqslant 2$.

Theorem 2.2 ([1, Theorem III.1.9]). Every splitting basic finite dimensional algebra with quiver $Q$ is isomorphic to a factor algebra $\mathbb{k} Q / I$, where $I$ is a regular ideal.

Corollary 2.3. For a splitting basic finite dimensional algebra $A$ there is a set of relations $\rho$ such that $A \cong \mathbb{k} Q(A) /\langle\rho\rangle$.

We now use Corollary 2.3 to get a concept of representation space that would be convenient for our purposes.

Let $A$ be a splitting (but not necessarily basic) finite dimensional algebra and $B$ be a maximal semisimple subalgebra in $A$ supplemented by the Jacobson radical $\mathfrak{r}$. Since $\mathbb{k}$ is algebraically closed, $B$ is isomorphic to $\prod_{i=1}^{s} \operatorname{Mat}_{n_{i} \times n_{i}}(\mathbb{k})$. We denote by $e_{j k}^{(i)}, i=1, \ldots, s, i, j=1, \ldots, n_{i}$, the elements of the canonical basis of $B$. We also denote by $a_{1}, \ldots, a_{q}$ the elements of $\bigcup_{i, j} e_{11}^{(i)} \mathfrak{r} e_{11}^{(j)}$ chosen in such a way that their residue classes give a basis for $\bigoplus_{i, j} e_{11}^{(i)} \mathfrak{r} / \mathfrak{r}^{2} e_{11}^{(j)}$. It is known that elements $e_{j k}^{(i)}$ and $a_{i}$ generate $A$ as an algebra.

Now fix a positive integer $d$. Then the set $\operatorname{Rep}(A, d)$ of all $d$-dimensional representations of $A$ is the set of all algebra homomorphisms $A \rightarrow \operatorname{Mat}_{d \times d}(\mathbb{k})$. Such a homomorphism is defined by the images of $e_{j k}^{(i)}$ and $a_{l}$. Thus $\operatorname{Rep}(A, d)$ may be identified with a Zariski closed subvariety in $\operatorname{Mat}_{d \times d}(\mathbb{k})\left(\sum_{i} n_{i}^{2}\right)+l$, where all the relations from $I$ are satisfied. The structure and properties of $\operatorname{Rep}(A, \alpha)$ as an algebraic variety are investigated in [2].

Observe that $e_{j j}^{(i)}$ are primitive pairwise orthogonal idempotents and $1=\sum e_{j j}^{(i)}$. So, we may define dimension vectors of $A$-modules according to this decomposition. For a module $M$ we set $(\underline{\operatorname{dim}}(M))_{i, j}:=\operatorname{dim}_{\mathbb{k}}\left(e_{j j}^{(i)} M\right)$. This identifies the representation space $\operatorname{Rep}(A, \alpha)$ with the subset in $\operatorname{Rep}\left(A, \sum_{i, j} \alpha_{i, j}\right)$, where each $e_{j j}^{(i)}$ is a projection onto an $\alpha_{i, j}$-dimensional subspace. On the representation space we have a natural action of $G L_{d}$, where $d=\sum_{i, j} \alpha_{i, j}$. It is easy to see that isomorphism classes of $\alpha$-dimensional $A$-modules coincide with $G L_{d}$-orbits in $\operatorname{Rep}(A, \alpha)$. 
Example 2.4. For a quiver $Q$ and a dimension vector $\alpha$ consider two representation spaces $\operatorname{Rep}(Q, \alpha)$ and $\operatorname{Rep}(\mathbb{k} Q, \alpha)$. The latter is larger because when we define $\operatorname{Rep}(Q, \alpha)$ we fix the projections $e_{i}, i \in Q_{0}$, and only vary the maps $a \in Q_{1}$.

In fact the information contained in $\operatorname{Rep}(A, \alpha)$ is excessive for the purposes of invariant theory. It is useful to consider the smaller subvariety that plays the same role as $\operatorname{Rep}(Q, \alpha)$ for $\operatorname{Rep}(\mathbb{k} Q, \alpha)$. Fix a decomposition $\mathbb{k}^{\sum_{i, j} \alpha_{i, j}}=\bigoplus_{i, j} V_{i, j}$, where the dimension of each $V_{i, j}$ equals $\alpha_{i, j}$. Define

$$
\underline{\operatorname{Rep}}(A, \alpha)=\left\{\left(x_{j, k}^{(i)}, y_{l}\right)_{i, j, k, l} \in \operatorname{Rep}(A, \alpha) \mid \operatorname{Im}\left(x_{j j}^{(i)}\right)=V_{i, j}, \text { for all } i, j\right\} .
$$

Observe that each $G L_{d}$-orbit in $\operatorname{Rep}(Q, \alpha)$ intersects $\operatorname{Rep}(A, \alpha)$ and moreover that the intersection of each $G L_{d}$-orbit with $\operatorname{Rep}(A, \alpha)$ is a single $G L(\alpha)$-orbit, where $G L(\alpha):=\prod_{i, j} G L\left(V_{i, j}\right)$. This implies that the moduli problem for $G L_{d}$-action on $\operatorname{Rep}(A, \alpha)$ may be reduced to the one for $G L(\alpha)$-action on $\operatorname{Rep}(A, \alpha)$.

If $A$ is the quotient algebra $\mathbb{k} Q /\langle\rho\rangle$, the set $\operatorname{Rep}(A, \alpha)$ embeds into $\operatorname{Rep}(Q, \alpha)$ as a Zariski closed subvariety, namely as the subset where $X_{p} \equiv 0$ for all $p \in\langle\rho\rangle$. Here for a representation $X \in \operatorname{Rep}(Q, \alpha)$ and an element

$$
\lambda_{1} a_{i_{11}} \ldots a_{i_{1 k(1)}}+\ldots+\lambda_{s} a_{i_{s 1}} \ldots a_{i_{s k(s)}}
$$

we denote by $X_{p}$ the linear transformation

$$
\lambda_{1} X_{a_{i_{11}}} \ldots X_{a_{i_{1 k(1)}}}+\ldots+\lambda_{s} X_{a_{i_{s 1}}} \ldots X_{a_{i_{s k(s)}}} .
$$

\section{Framed Representations of Finite dimensional algebras}

3.1. Framed representations. Let $A$ be a (splitting basic) finite dimensional algebra with $\left|Q(A)_{0}\right|=n$. Fix two dimension vectors $\alpha, \zeta \in\left(\mathbb{Z}_{\geqslant 0}\right)^{n}$ and a $Q(A)_{0^{-}}$ graded vector space $V=V_{1} \oplus \ldots \oplus V_{n}$ such that $\operatorname{dim} V_{i}=\zeta_{i}$, for $i=1, \ldots, n$. Consider the extended representation space $\operatorname{Rep}(A, \alpha, \zeta)$ consisting of pairs $(M, \tilde{f})$ with $M \in \operatorname{Rep}(A, \alpha)$ and $\widetilde{f}=\left(\widetilde{f}_{i}: M \rightarrow V_{i}\right)_{i=1}^{n}$ as a tuple of linear maps such that $\widetilde{f}_{i} e_{i}=\widetilde{f}_{i}$, for all $i \in Q_{0}$ and $\widetilde{f}_{i} e_{j}=0$, for $j \neq i$. The latter two conditions mean that each $\tilde{f}_{i}$ factors through the projection of $M$ onto $e_{i} M$ along $\bigoplus_{i \neq j} e_{j} M$. Elements of $\operatorname{Rep}(A, \alpha, \zeta)$ will be called framed representations of the algebra $A$. Define the action of $G L(\alpha)$ on this variety by $g \cdot\left(M,\left(f_{i}\right)_{i=1}^{n}\right)=\left(g \cdot M,\left(f_{i} g^{-1}\right)_{i=1}^{n}\right)$.

When passing from $\operatorname{Rep}(A, \alpha)$ to $\operatorname{Rep}(A, \alpha)$ we fix the spaces $e_{i} M$, so we may actually replace $\widetilde{f}_{i}$ by $f_{i}=\left.\widetilde{f}_{i}\right|_{e_{i} M}$. Thus we obtain the simplified version of $\operatorname{Rep}(A, \alpha, \zeta)$ as $\operatorname{Rep}(A, \alpha, \zeta)=\left\{(M, f) \mid M \in \operatorname{Rep}(A, \alpha), f=\left(f_{i}\right)_{i=1}^{n}: M_{1} \oplus \ldots \oplus\right.$ $M_{n} \rightarrow V_{1} \oplus \ldots \overline{\oplus V}_{n}$ is a graded linear map $\} \cong \operatorname{Rep}(A, \alpha) \oplus \bigoplus_{i=1}^{n} \operatorname{Hom}_{\mathbb{k}}\left(\mathbb{k}^{\alpha_{i}}, V_{i}\right)$, where $\operatorname{dim} V_{i}=\zeta_{i}$. The $G L_{d}$-action on $\operatorname{Rep}(A, \alpha, \bar{\zeta})$ restricts to the following action of $G L(\alpha)$ on $\underline{\operatorname{Rep}}(A, \alpha, \zeta): g \cdot\left(M,\left(f_{i}\right)_{i=1}^{n}\right)=\left(g \cdot M,\left(f_{i} g_{i}^{-1}\right)_{i=1}^{n}\right)$.

Definition 3.1. A pair $(M, f) \in \operatorname{Rep}(A, \alpha, \zeta)$ is stable if no nonzero submodule of $M$ is contained in ker $f$. The set consisting of such pairs will be denoted by $\operatorname{Rep}^{s}(A, \alpha, \zeta)$. We will also denote by $\operatorname{Rep}^{s}(A, \alpha, \zeta)$ the subset of all stable pairs in $\underline{\operatorname{Rep}}(A, \alpha, \zeta)$.

It is easy to see that the thus constructed framed representation space $\underline{\operatorname{Rep}}(A, \alpha, \zeta)$ embeds in $\operatorname{Rep}(Q, \alpha, \zeta)$ as a Zariski closed $G L(\alpha)$-invariant subvariety. It is a general fact of invariant theory (see [10, $\S I I .3 .2])$ that in this case the restriction $\pi_{A}$ of the quotient map $\pi: \operatorname{Rep}^{s}(Q, \alpha, \zeta) \rightarrow \mathcal{M}(Q, \alpha, \zeta)$ to $\underline{\operatorname{Rep}}^{s}(A, \alpha, \zeta)$ 
is again a quotient map. Moreover, the restriction of the natural morphism $\mathcal{M}^{s}(Q, \alpha, \zeta) \rightarrow \mathcal{M}(Q, \alpha, \zeta)=\mathcal{M}(Q, \alpha)$ to $\mathcal{M}^{s}(A, \alpha, \zeta)$ gives us a projective morphism $\mathcal{M}^{s}(A, \alpha, \zeta) \rightarrow \mathcal{M}(A, \alpha)=\{p t\}$. Collecting these observations and the well-known properties of moduli spaces for quivers, we obtain the following.

Proposition 3.2. The quotient $\pi_{A}: \operatorname{Rep}^{s}(A, \alpha, \zeta) \rightarrow \mathcal{M}^{s}(A, \alpha, \zeta)$ is geometric. Moreover, if $\operatorname{Rep}^{s}(A, \alpha, \zeta)$ is nonempty, then $\mathcal{M}^{s}(A, \alpha, \zeta)$ is a smooth projective variety.

3.2. The construction of the quotient space. From now on for $\sigma \in \mathbb{k} Q$ we will denote by $\bar{\sigma}$ its image $\sigma+\langle\rho\rangle$ in $\mathbb{k} Q /\langle\rho\rangle \cong A$. It is easy to see that in $\mathbb{k} Q /\langle\rho\rangle$ there is a (finite) $\mathbb{k}$-basis $\Xi$ consisting of images of paths in $\mathbb{k} Q$. Its elements will be referred to as paths in the algebra $A$. Denote by $I_{i}$ the injective $A$-module associated with the $i$-th vertex of the quiver. Recall that the corresponding representation from $\underline{\operatorname{Rep}}(Q, \rho)$ may be described as follows: $\left(I_{i}\right)_{j}=\operatorname{span}\{\bar{\tau}: j \rightsquigarrow i\}^{*}$, where " $\bar{\tau}: j \rightsquigarrow i "$ means that $\bar{\tau}$ is the image of a path $\tau$ starting in the $j$-th vertex and ending in the $i$-th one. In this case $\left(\left(I_{i}\right)_{a: k \rightarrow l} f\right)(\bar{\tau})=f(\bar{\tau} \bar{a})$, where $\bar{\tau}: l \rightsquigarrow i$. Consider the injective module $J:=\bigoplus_{i \in Q_{0}} I_{i} \otimes_{\mathbb{k}} V_{i}$. Observe that as a $\mathbb{k}$-linear space

$$
J_{i}=e_{i} J \cong \bigoplus_{j \in Q_{0}}\left(I_{j}\right)_{i} \otimes_{\mathbb{k}} V_{j} \cong \bigoplus_{j \in Q_{0}} \bigoplus_{\Xi \ni \bar{\tau}: i \rightsquigarrow j} V_{j} \cong \bigoplus_{\Xi \ni \bar{\tau}: i \rightsquigarrow j} V_{j}
$$

Now, given a point $(M, f) \in \underline{\operatorname{Rep}}(A, \alpha, \zeta)$, define a map $\Phi_{(M, f)}=\left(\varphi_{i}\right)_{i \in Q_{0}}$ : $M \rightarrow J$ by the following rule:

$$
\varphi_{i}=\bigoplus_{\Xi \ni \bar{\tau}: i \rightsquigarrow j} f_{j} \bar{\tau}: M_{i} \rightarrow \bigoplus_{\Xi \ni \bar{\tau}: i \rightsquigarrow j} V_{j} .
$$

Here we view $\bar{\tau}$ as an element of $A$; i.e. $\bar{\tau}(m)=\bar{\tau} \cdot m$.

Proposition 3.3. The subspace $\operatorname{ker} \Phi_{(M, f)}=\bigoplus_{i \in Q_{0}} \operatorname{ker} \varphi_{i}$ is the maximal $A$ submodule of $M$ contained in $\operatorname{ker} f$.

Proof. It is a straightforward computation to check that $\Phi_{(M, f)}$ is a homomorphism of $A$-modules. Hence $\operatorname{ker} \Phi_{(M, f)}$ is an $A$-submodule of $M$. Now let $U$ be an $A$ submodule of $M$ contained in ker $f$. For each $\Xi \ni \bar{\tau}: i \rightsquigarrow j$ we then have $\bar{\tau} U_{i}=$ $\bar{\tau} e_{i} U=\bar{\tau} U=e_{j} \bar{\tau} U \subseteq U_{j}$. This implies that $f_{j}(\bar{\tau} \cdot x)=0$, for all $x \in U, j \in Q_{0}, \bar{\tau} \in$ $\Xi$, i.e. $U \subseteq \operatorname{ker} \Phi_{(M, f)}$.

Corollary 3.4. The map $\Phi_{(M, f)}: M \rightarrow J$ is injective if and only if the pair $(M, f)$ is stable.

Now introduce the notion of the Grassmannian of submodules. Let $N=\bigoplus_{i \in Q_{0}} N_{i}$ be an $A$-module; the Grassmannian of $A$-submodules of $N$ with dimension vector $\gamma$ is a set $\operatorname{Gr}_{\gamma}^{A}(N)$ of all $Q_{0}$-graded subspaces $U=\bigoplus_{i \in Q_{0}} U_{i} \subseteq N$ with $\operatorname{dim} U_{i}=\gamma_{i}$ that are $A$-submodules. Note that $\operatorname{Gr}_{\gamma}^{A}(N)$ is a closed variety in the product of classical Grassmannians $\operatorname{Gr}_{\gamma}(N)=\prod_{i \in Q_{0}} \operatorname{Gr}_{\gamma_{i}}\left(N_{i}\right)$ defined by $\bar{\tau}\left(U_{i}\right) \subseteq U_{j}$, for all $\Xi \ni \bar{\tau}: i \rightsquigarrow j$.

The following result is a generalization of [13, Proposition 3.9].

Theorem 3.5. The moduli space $\mathcal{M}^{s}(A, \alpha, \zeta)$ is isomorphic to the Grassmannian of submodules $\operatorname{Gr}_{\alpha}^{A}(J)$. 
Proof. Denote by $\operatorname{IHom}_{\alpha}(V)$ the set of all injective graded vector space homomorphisms from a space with dimension vector $\alpha$ to $V$. It is easy to see that $\operatorname{Gr}_{\alpha}(J)$ is a quotient of $\prod_{i \in Q_{0}} \operatorname{IHom}_{\mathbb{k}}\left(M_{i}, J_{i}\right)$ by the natural action of $G L(\alpha)$ (note that $G L\left(\alpha_{i}\right)$ acts on $M_{i}$ by base change). Denote the inverse image of $\operatorname{Gr}_{\alpha}^{A}(J) \subseteq \operatorname{Gr}_{\alpha}(J)$ by $\operatorname{IHom}_{\alpha}^{A}(J)$. Now to prove the theorem it is sufficient to construct a $G L(\alpha)$-invariant isomorphism $\Phi: \underline{\operatorname{Rep}}(A, \alpha, \zeta) \stackrel{\sim}{\rightarrow} \operatorname{IHom}_{\alpha}^{A}(J)$. We set $\Phi:(M, f) \mapsto \Phi_{(M, f)}$. This map is $G L(\alpha)$-invariant. Indeed, let $g=\left(g_{i}\right)_{i \in Q_{0}} \in G L(\alpha)$. Then for $m \in M$ we have $\Phi(g \cdot(M, f))_{i}\left(m_{i}\right)=\left(\Phi_{g \cdot(M, f)}\right)_{i}(m)=\bigoplus_{\Xi \ni \bar{\tau}: i \rightsquigarrow j} f_{j}\left(g_{j} \bar{\tau} m\right)=\left(g \cdot \Phi_{(M, f)}\right)(m)$.

From Corollary 3.4 it follows that $\Phi\left(\underline{\operatorname{Rep}}^{s}(A, \alpha, \zeta)\right) \subseteq \operatorname{IHom}_{\alpha}^{A}(J)$. Let us show that given an injection $\{F: M \hookrightarrow J\} \in \overline{\operatorname{IHom}}_{\alpha}^{A}(J)$, the pair $(M, f)$ may be recovered. But $f$ is obtained as a composition

$$
f: M_{i} \stackrel{\varphi_{i}}{\longrightarrow} \bigoplus_{\Xi \ni \bar{\tau}: i \rightsquigarrow j} V_{j} \rightarrow V_{i},
$$

where the last map is a projection on a summand associated to $\bar{\tau}=e_{i}$. As for the module $M$, the following lemma gives the possibility to recover it.

Lemma 3.6. Let $K$ be a finite dimensional submodule in $J$ and $\operatorname{Rep}^{s}(A, \alpha, K) \subseteq$ $\operatorname{Rep}^{s}(A, \alpha)$ be the inverse image of $\operatorname{IHom}_{\alpha}^{A}(K)$ under $\Phi$. Then there is a morphism

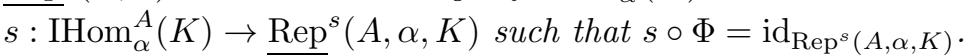

Proof. To recover $M \in \operatorname{Rep}^{s}(A, \alpha, K)$ means to define the action of $A$ on the vector space $\bigoplus_{i \in Q_{0}} M_{i}$, i.e. of the elements $A \ni \bar{a}: i \rightarrow j$ for $a \in Q_{1}$. Furthermore, for each of them we have the following commutative diagram:

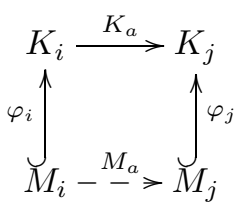

where $M_{a}=\left.\bar{a}\right|_{M_{i}}$. This is because for each pair $(M, f)$ the map $\Phi_{(M, f)}$ is an $A$ homomorphism. So, each $M_{a}$ satisfies the equations $K_{a} \varphi_{i}=\varphi_{j} M_{a}$, which may be considered as matrix equations. Analogously, $\varphi_{j}$ may be regarded as a matrix of dimension $\operatorname{dim} K_{j} \times \operatorname{dim} M_{j}$. Its rank is maximal and equals $\operatorname{dim} M_{j}$, since $\varphi_{j}$ are injections. Therefore, $\operatorname{IHom}_{\alpha}^{A}(K)$ may be covered by open subsets, where various minors of the matrix of $\varphi_{j}$ do not vanish, and $M_{a}$ are recovered from matrix elements of $\varphi_{i}, \varphi_{j}$ and $K_{a}$ using Cramer's Theorem.

Together with the above described way of recovering $f$, the morphism

$$
s: \operatorname{IHom}_{\alpha}^{A}(J) \rightarrow \underline{\operatorname{Rep}}^{s}(A, \alpha)
$$

gives a morphism that is inverse to $\Phi$. Consequently, $\Phi$ is an isomorphism and, being $G L(\alpha)$-invariant, descends to quotients implying that $\Phi / / G L(\alpha): \mathcal{M}^{s}(A, \alpha, \zeta) \cong$ $\underline{\operatorname{Rep}}^{s}(A, \alpha, \zeta) / / G L(\alpha) \stackrel{\sim}{\longrightarrow} \operatorname{IHom}_{\alpha}^{A}(J) / / G L(\alpha) \cong \operatorname{Gr}_{\alpha}^{A}(J)$. Theorem 3.5 is proved.

Theorem 3.5 describes a variety that may serve as a substitute of a moduli space of $A$-modules with dimension vector $\alpha$ whenever $\operatorname{Rep}^{s}(A, \alpha, \zeta)$ is nonempty. So, it is important to have a criterion of existence of a stable pair. For quivers M. Reineke proved that $\operatorname{Rep}^{s}(A, \alpha, \zeta) \neq \varnothing$ if and only if $\zeta_{i} \geqslant\left(\mathbf{i}, \alpha_{i}\right)_{Q}$, for all $i \in Q_{0}$, where i stands for the vector with all coordinates zero except 1 on the $i$-th place, and 
$(\cdot, \cdot)_{Q}$ is the Euler form, i.e. $(\mathbf{i}, \mathbf{j})_{Q}=\delta_{i j}-($ number of arrows from $i$ to $j$ ); see [13, Proposition 4.3]. For arbitrary finite dimensional algebras we do not have such a result. However, we can state a weaker proposition. Recall that a socle of an $A$-module $M$ is the sum of all its simple submodules.

Proposition 3.7. For an A-module $M$ with dimension vector $\alpha$ there is a map $f: M \rightarrow V$ making the pair $(M, f) \in \operatorname{Rep}(A, \alpha, \zeta)$ stable if and only if the socle soc $M$ may be embedded in $\operatorname{soc} J$.

Proof. The "only if" part is trivial. We now prove sufficiency. First, note that $(\operatorname{soc} J)_{i}=V_{i}^{e_{i}}$. Consider a decomposition $M=\operatorname{soc} M \oplus W$ of $M$ as a vector space. Let $f$ be the composition of the projection of $M$ onto soc $M$ along $W$ and an inclusion $\operatorname{soc} M \hookrightarrow \operatorname{soc} J=\bigoplus_{i \in Q_{0}} V_{i}$. Then ker $f=W$. Now, if $W$ contains a submodule $N$, then $W \supseteq \operatorname{soc} N$, which is a contradiction $\operatorname{since} \operatorname{soc} N \subseteq \operatorname{soc} M$.

Taking the dimensions of $(\operatorname{soc} J)_{i}$ and $(\operatorname{soc} M)_{i}$, his condition may be reformulated as: for $M \in \operatorname{Rep}(Q, \alpha)$ there is a map $f: M \rightarrow V$ making the pair $(M, f) \in \operatorname{Rep}(A, \alpha, \zeta)$ stable if and only if $\zeta_{i} \geqslant \operatorname{dim}(\operatorname{soc} M)_{i}$. Note that $(\operatorname{soc} M)_{i}$ is the multiplicity in $\operatorname{soc} M$ of the simple module $S(i)=\mathbb{k} e_{i}$ corresponding to the $i$-th vertex. For quivers this obviously coincides with [13, Lemma 4.1]. When using this criterion it is also convenient to have in mind that $(\operatorname{soc} M)_{i}=\bigcap_{\bar{a}: i \rightarrow j} \operatorname{ker} M_{\bar{a}}$.

Now, we need a way of determining, for a point of the classical Grassmannian $\operatorname{Gr}_{\alpha}(J)=\prod_{i \in Q_{0}} \operatorname{Gr}_{\alpha_{i}}\left(J_{i}\right)$, whether it lies in the Grassmanian of submodules. To formulate the next proposition, we should recall that the summands $V_{s}$ of $J_{i}$ are indexed by paths in $A$. Furthermore, since an arrow $\bar{a}: i \rightarrow s$ induces, for each $V_{k}^{(\bar{\tau})} \subseteq J_{i}$ with $\bar{\tau}=\bar{\tau}^{\prime} \bar{a}: i \rightsquigarrow k$, an isomorphism $V_{k}^{(\bar{\tau})} \stackrel{\sim}{\longrightarrow} V_{k}^{\left(\bar{\tau}^{\prime}\right)}$, there is an injection $\dagger_{\bar{a}}: \widetilde{J}_{s}^{(\bar{a})} \hookrightarrow J_{i}$, where $\widetilde{J}_{s}^{(\bar{a})}$ is a sum of all $V_{k}^{(\bar{\sigma})} \subseteq J_{s}$ such that $\overline{\sigma a} \neq 0$. This injection acts as a simple index change, and, moreover, $\bar{a} \dagger_{\bar{a}}=\mathrm{id}{\widetilde{J_{s}}}_{(\bar{a})}$. Note that for hereditary algebras, i.e. for quivers with no relations, $\widetilde{J}$ coincides with $J$, so $J_{s}$ is embedded in $J_{i}$ whenever there is an arrow $i \rightarrow s$.

Proposition 3.8. A point $\left(U_{i} \subseteq \bigoplus_{\Xi \ni \bar{\tau} i \rightsquigarrow j} V_{j}\right)_{i \in Q_{0}} \in \prod_{i \in Q_{0}} \operatorname{Gr}_{\alpha_{i}}\left(\bigoplus_{\Xi \ni \bar{\tau}: i \rightsquigarrow j} V_{j}\right)$ lies in the image of $\Phi / / G L(\alpha)$ if and only if

$$
U_{i} \subseteq V_{i}^{\left(e_{i}\right)} \oplus \bigoplus_{T(\bar{a}) \ni \bar{\tau}: k \rightsquigarrow l} V_{l}^{(\bar{\tau})} \oplus \dagger_{\bar{a}}\left(U_{j} \cap \widetilde{J}_{j}^{(\bar{a})}\right), \quad \forall i \in Q_{0}, \bar{a}: i \rightarrow j,
$$

where $T(\bar{a})$ is the set of all $\Xi \ni \bar{\tau}: k \rightsquigarrow l$ such that there is an arrow $\bar{a} \neq \bar{b}: i \rightarrow k$ and there is no path $\sigma$ in $Q$ with $\bar{\tau} \bar{b}=\overline{\sigma a}$.

Proof. As it was shown above, $\left(U_{i} \subseteq \bigoplus_{\Xi \ni \bar{\tau} i \rightsquigarrow j} V_{j}\right)_{i \in Q_{0}} \in \prod_{i \in Q_{0}} \operatorname{Gr}_{\alpha_{i}}\left(\bigoplus_{\Xi \ni \bar{\tau}: i \rightsquigarrow j} V_{j}\right)$ belongs to the image of $\Phi / / G L(\alpha)$ if and only if it is an $A$-submodule of $J$, which means that $\bar{\tau}\left(U_{i}\right) \subseteq U_{j}, \forall \Xi \ni \bar{\tau}: i \rightarrow j$. It is straightforward to check that these conditions are equivalent to $(3)$.

On $\mathcal{M}^{s}(A, \alpha, \zeta)$ one may construct the universal family $\mathcal{C}=\left(\left(\mathcal{C}_{i}\right)_{i \in Q_{0}},\left(\mathcal{C}_{a}\right)_{a \in Q_{0}}\right)$. For a point $y=\left(U_{i} \subseteq J_{i}\right)_{i \in Q_{0}}$, we take $\left(\mathcal{C}_{i}\right)_{y}=\operatorname{pr}_{i}\left(U_{i}\right)$, where $\operatorname{pr}_{i}$ is the projection of $\bigoplus_{\bar{\tau}: i \rightsquigarrow j} V_{j}^{(\bar{\tau})}$ onto $V_{i}^{\left(e_{i}\right)}$, and $\left(\mathcal{C}_{a}\right)_{y}:\left(\mathcal{C}_{t a}\right)_{y} \rightarrow\left(\mathcal{C}_{h a}\right)_{y}$ acts as $J_{a}$. It is not hard to check that the maps $\mathcal{C}_{a}$ are well defined.

Now let $Q$ be a quiver without oriented cycles and $i_{0} \in Q_{0}$ be a source. Also let $Q^{\prime}$ be a full subquiver of $Q$ with support $Q_{0} \backslash\left\{i_{0}\right\}$. Denote by $\alpha^{\prime}$ and $\zeta^{\prime}$ the 
restrictions of $\alpha$ and $\zeta$ respectively to $Q^{\prime}$. Notice that $I=\operatorname{span}\left\{\bar{\tau}: i_{0} \rightsquigarrow j\right\}$ is a two-sided ideal in $A$, so we may consider a factor algebra $A^{\prime}=A / I$. Clearly it may be associated with a subalgebra of $A$ generated by $e_{i}$, for $i \neq i_{0}$, and $a \in Q_{1}$ with $t a \neq i_{0}$. Then the subspace $J^{\prime}=\bigoplus_{i \in Q_{0}^{\prime}} J_{i} \subseteq J$ is naturally an injective $A^{\prime}$-module. So, $\operatorname{Gr}_{\alpha}^{A}(J)$ is a subvariety in $\operatorname{Gr}_{\alpha^{\prime}}^{A^{\prime}}\left(J^{\prime}\right) \times \operatorname{Gr}_{\alpha_{i_{0}}}\left(\bigoplus_{\bar{\tau}: i \rightsquigarrow j} V_{j}^{(\tau)}\right)$ consisting of pairs $\left(\left(U_{i}\right)_{i \in Q_{0}^{\prime}}, U\right)$ satisfying conditions from Proposition 3.8. If there are no relations starting from $i$, by [13, Lemma 4.9] the projection $\operatorname{Gr}_{\alpha}^{A}(J) \rightarrow \operatorname{Gr}_{\alpha^{\prime}}^{A^{\prime}}\left(J^{\prime}\right)$ is isomorphic to the Grassmann bundle $\operatorname{Gr}_{\alpha_{0}}\left(V_{i} \oplus \bigoplus_{a: i \rightarrow j} \mathcal{C}_{j}\right)$. Therefore, in this case a reduction similar to the one introduced in [13, Section 4.3] may be performed.

Example 3.9. Let $A$ be the algebra with quiver $Q: 1 \stackrel{a_{1}}{\longrightarrow} 2 \stackrel{a_{2}}{\longrightarrow} 3$ and relations $\rho=\left\{a_{2} a_{1}\right\}$. So, $A$ is a Nakayama algebra with admissible sequence $(1,1)$; see [1, Section IV.2]. Consider two dimension vectors $\alpha$ and $\zeta$ and a vector space $V_{i}$ for each $\zeta_{i}$. Then, as we have proved, $\mathcal{M}^{s}(A, \alpha, \zeta) \cong \operatorname{Gr}_{\alpha}^{A}(J)$, where $J_{1}=V_{1} \oplus V_{2}, J_{2}=V_{2} \oplus V_{3}$, and $J_{3}=V_{3}$. Using Proposition 3.8 we obtain that $\mathcal{M}^{s}(A, \alpha, \zeta) \cong\left\{\left(U_{i} \subseteq J_{i}\right)_{i=1}^{3} \mid U_{3} \subseteq V_{3}, U_{2} \subseteq V_{2} \oplus U_{3}, U_{1} \subseteq V_{1} \oplus\left(U_{2} \cap V_{2}\right)\right\}$. Note that in this example we do not need to write the index $\left(e_{i}\right)$ over $V_{i}$, since there is only one summand $V_{i}$ in $J_{i}$.

Example 3.10. Let $A$ be the algebra with quiver

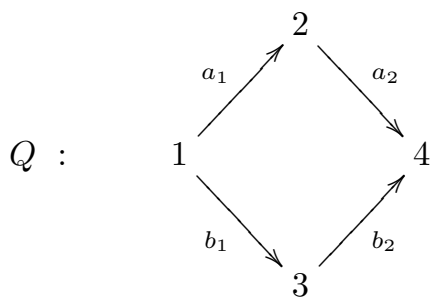

and a single relation $a_{2} a_{1}-b_{2} b_{1}$. Then $A$ is a $\mathbb{k}$-linear span of the path images $\bar{e}_{i}, i=1, \ldots, 4, \bar{a}_{i}, \bar{b}_{i}$ and $\bar{a}_{2} \bar{a}_{1}$. The components of $J$ are $J_{1}=V_{1} \oplus V_{2} \oplus V_{3} \oplus$ $V_{4}, J_{2}=V_{2} \oplus V_{4}, J_{3}=V_{3} \oplus V_{4}$ and $J_{4}=V_{4}$ with the arrow images acting as $\bar{a}_{1}=0 \oplus \mathrm{id} \oplus 0 \oplus \mathrm{id}, \bar{b}_{1}=0 \oplus 0 \oplus \mathrm{id} \oplus \mathrm{id}, \bar{a}_{2}=0 \oplus \mathrm{id}, \bar{a}_{4}=0 \oplus \mathrm{id}$. Hence $\widetilde{J}_{i}=J_{i}$, for all $i$, and all †'s are natural inclusions. That is why we venture to omit them as well as the indexes $\left(e_{i}\right)$ in the final formula. So, $\mathcal{M}^{s}(A, \alpha, \zeta) \cong$ $\left\{\left(U_{i} \subseteq J_{i}\right)_{i=1}^{4} \mid U_{1} \subseteq V_{1} \oplus V_{3} \oplus U_{2}, U_{1} \subseteq V_{1} \oplus V_{2} \oplus U_{3}, U_{2} \subseteq V_{2} \oplus U_{4}, U_{3} \subseteq V_{3} \oplus U_{4}\right\}$.

Example 3.11. Consider the algebra $A=\mathbb{k}[x] /(x)^{n}$. It is also a Nakayama algebra with admissible sequence $(n)$, but now its quiver contains a loop. In fact, it is a Jordan quiver with a single vertex and a single loop $a$, and the only relation is $a^{n}$. Let $\alpha=(m), \zeta=(k)$, and $V$ be a $k$-dimensional vector space. Then $J=J_{1}=V^{(e)} \oplus V^{(a)} \oplus \ldots \oplus V^{\left(a^{n-1}\right)}$. Observe that $\bar{a}$ acts on $J$ as follows:

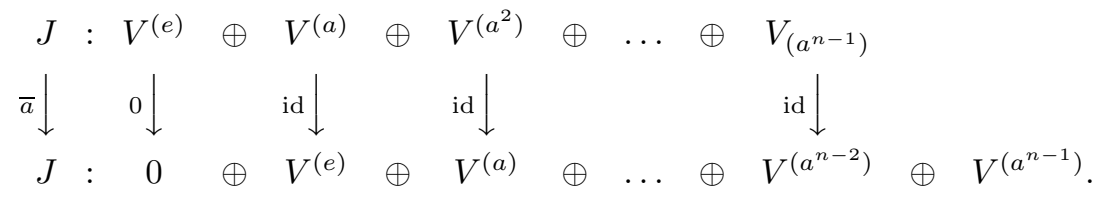

So, $\widetilde{J}=V^{(e)} \oplus V^{(a)} \oplus \ldots \oplus V^{\left(a^{n-2}\right)}$, and $\dagger_{\bar{a}} \operatorname{maps} V^{\left(a^{t}\right)}, t=0, \ldots, n-2$, to $V^{\left(a^{t+1}\right)}$ isomorphically. 
It is easy to check that $J$ can be viewed as $\mathbb{k}[x] /\left(x^{n}\right) \otimes V$ with $\bar{a}$ acting as $\frac{d}{d t}$. Then $\widetilde{J}$ becomes $\mathbb{k}[x] /\left(x^{n-1}\right) \otimes V$, $\dagger_{\bar{a}}: f \otimes v \mapsto x f \otimes v$, and the moduli space is isomorphic to the Grassmannian of $m$-dimensional $\frac{d}{d t}$-invariant subspaces in $J$. This interpretation is in fact rather fruitful and will be further explored in the next sections.

Denote by $\mathbb{k} Q^{(1)}$ an ideal in the path algebra generated by all oriented cycles in $Q$. We also use the notation $\mathbb{k} Q^{(t)}:=\left(\mathbb{k} Q^{(1)}\right)^{t}$.

Let $G$ be a reductive group acting on a vector space $X$. Recall that the null cone of this action is the set $\mathcal{N}=\{x \in X \mid f(x)=0$, for all nonconstant homogeneous $G$-invariant functions on $X\}$. By Hilbert-Mumford's Criterion [10, Theorem III.2.4] this is equivalent, for $x \in X$, to the existence of a one-parameter subgroup $\lambda$ : $\mathbb{k} \backslash\{0\} \rightarrow G$ with $\lim _{t \rightarrow 0} \lambda(t) x=0$.

One of the possible applications of the above construction is the study of the null cone of $\operatorname{Rep}(Q, \alpha, \zeta)$ in the case when $Q$ is a quiver with oriented cycles. This is made possible by the following proposition.

Proposition 3.12. Let $Q$ be a quiver with oriented cycles. Then the null cone of the variety $\operatorname{Rep}(Q, \alpha, \zeta)$ is isomorphic to $\operatorname{Rep}\left(\mathbb{k} Q /(\mathbb{k} Q)^{\left(\max _{i} \alpha_{i}\right)}, \alpha, \zeta\right)$.

Proof. First of all, recall what is the categorical quotient of a framed representation space. There is a $G L(\alpha)$-invariant isomorphism $\psi: \operatorname{Rep}(Q, \alpha, \zeta) \cong \operatorname{Rep}(\widetilde{Q}, \widetilde{\alpha})$ for a quiver $\widetilde{Q}$ and an extended dimension vector $\widetilde{\alpha}$ (see [13]). We have

$$
\operatorname{Rep}(Q, \alpha, \zeta) / / G L(\alpha) \cong \operatorname{Rep}(\widetilde{Q}, \widetilde{\alpha}) / / G L(\alpha)
$$

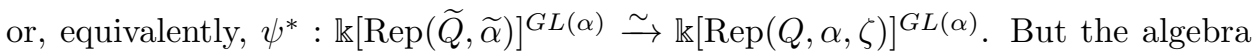
$\mathbb{k}[\operatorname{Rep}(\widetilde{Q}, \widetilde{\alpha})]^{G L(\alpha)}$ is generated by the traces of oriented cycles in $\widetilde{Q}$. On the other hand, oriented cycles in $\widetilde{Q}$ are those of $Q$, and hence the algebra $\mathbb{k}[\operatorname{Rep}(Q, \alpha, \zeta)]^{G L(\alpha)}$ is also generated by traces of the oriented cycles in $Q$.

Thus the null cone consists of pairs $(M, f)$, where $M$ are such representations on which all the oriented cycles in $Q$ act as nilpotent operators. Since $A^{k}=0$ for a nilpotent operator $A$ in a $k$-dimensional space, all the oriented cycles as operators in $M$ vanish in the $\left(\max _{i} \alpha_{i}\right)$-th power. But we state that the stronger property holds: the product of any $\max _{i} \alpha_{i}$ oriented cycles is zero. If $\sigma_{1}$ and $\sigma_{2}$ are two such cycles, then by our conditions $\sigma_{1} \sigma_{2}$ and $\sigma_{2} \sigma_{1}$, as well as any of their product, are nilpotent. Moreover, all of them vanish in the same power. This implies that the commutator $\left[\sigma_{1}, \sigma_{2}\right]$ is also nilpotent. Indeed, any of its power equals a sum of products of $\sigma_{i}$. Since these products are nilpotent, their traces are all zero, providing that the trace of any power of the operator $\left[\sigma_{1}, \sigma_{2}\right]$ is zero. Therefore, the commutator $\left[\sigma_{1}, \sigma_{2}\right]$ is nilpotent.

Now, using Engel's Theorem, we can conclude that there is such a basis in the representation space where the matrices of both $\sigma_{1}$ and $\sigma_{2}$ are upper-triagonal with zeroes on the diagonal. Applying induction, we get a basis in which the matrices of all oriented cycles starting in a given vertex are upper-niltriagonal. As a product of oriented cycles starting in different vertices is zero by definition, the product of any $\max _{i} \alpha_{i}$ such operators is zero.

We briefly discuss an application of this construction. Let $\mathbb{k}$ be field $\mathbb{C}$ of complex numbers. Since $\operatorname{Rep}^{s}(Q, \alpha, \zeta) / / G L(\alpha)$ is projective over $\operatorname{Rep}(Q, \alpha, \zeta) / / G L(\alpha)=$ $\operatorname{Rep}(Q, \alpha) / / G L(\alpha)$ (see [9]), there is a natural projection $\pi_{s}: \operatorname{Rep}^{s}(Q, \alpha, \zeta) / / G L(\alpha)$ 
$\rightarrow \operatorname{Rep}(Q, \alpha) / / G L(\alpha)$. From [6, Theorem 4.1] we know that for each point $y \in$ $\operatorname{Rep}(Q, \alpha) / / G L(\alpha)$ there is a quiver $\widetilde{Q}$ and a pair of dimension vectors $\bar{\alpha}, \bar{\zeta} \in \mathbb{Z}_{\geqslant 0}^{\widetilde{Q}_{0}}$ such that $\pi_{s}^{-1}(y) \cong \widetilde{\pi}_{s}^{-1}(0)$, where $\widetilde{\pi}_{s}$ is the natural projection $\operatorname{Rep}^{s}(\widetilde{Q}, \widetilde{\alpha}, \widetilde{\zeta}) / / G L(\widetilde{\alpha})$ $\rightarrow \operatorname{Rep}(\widetilde{Q}, \widetilde{\alpha}) / / G L(\widetilde{\alpha})$. As a consequence, we obtain

Corollary 3.13. If $\mathbb{k}=\mathbb{C}$, then for every point $y \in \operatorname{Rep}(Q, \alpha) / / G L(\alpha)$ there is a finite dimensional algebra $A$, an injective $A$-module $J$ and a dimension vector $\beta \in \mathbb{Z}^{Q(A)_{0}}$ such that $\pi_{s}^{-1}(y) \cong \operatorname{Gr}_{\beta}^{A}(J)$.

As an example we work out the cyclic quiver $Q$ of type $A_{n-1}^{(1)}$ :

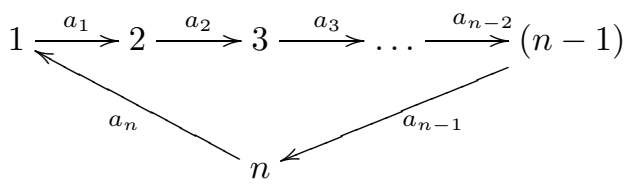

It is well known that points of $\operatorname{Rep}(Q, \alpha) / / G L(\alpha)$ are in one-to-one correspondence with isomorphism classes of semisimple representations of $Q$. For the quiver, the considered simple representations are the ones from the following list:

- $S_{i}$, for $i \in Q_{0}$, with $\operatorname{dim}\left(S_{i}\right)_{j}=\delta_{i j}$ and

- $E_{\lambda}$, for $\lambda \in \mathbb{k} \backslash\{0\}$, with dimension vector $(1, \ldots, 1)$ having $a_{1}=\ldots=$ $a_{n-1}=1$, and $a_{n}=\lambda$.

Choose an isomorphism class $y:=\left[\bigoplus_{i=1}^{n} m_{i} S_{i} \oplus \bigoplus_{\lambda \in \Lambda} m_{\lambda} E_{\lambda}\right]$. The new quiver from [6, Theorem 4.1] is then the disjoint union of $Q$ (if some of $m_{i}$ is nonzero) and $|\Lambda|$ copies of $A_{0}^{(1)}$. Moreover, let $\widehat{\pi}_{m_{\lambda}}, \lambda \in \Lambda$, be the projection

$$
\operatorname{Rep}^{s}\left(A_{0}^{(1)}, m_{\lambda}, \sum_{i \in Q_{0}} \zeta_{i}\right) / / G L(\alpha) \rightarrow \operatorname{Rep}\left(A^{(1)}, m_{\lambda}\right) / / G L(\alpha)
$$

and $\widehat{\pi}_{s}$ be the projection $\operatorname{Rep}^{s}(Q, \underline{m}, \zeta) / / G L(\alpha) \rightarrow \operatorname{Rep}(Q, \underline{m}) / / G L(\alpha)$, where $\underline{m}=$ $\left(m_{1}, \ldots, m_{n}\right)$. Then we have $\pi_{s}^{-1}(y) \cong \widehat{\pi}_{s}^{-1}(0) \times \prod_{\lambda \in \Lambda} \widehat{\pi}_{m_{\lambda}}^{-1}(0)$ (the first factor would be absent if all $\left.m_{i}=0\right)$. For $\widehat{\pi}_{m_{\lambda}}^{-1}(0)$ we may now apply considerations outlined in Example 3.11. In Section 5 we will give an explicit realization of the fibers by equations in projective space along with its version for $\mathbb{k}=\mathbb{R}$. In Section 4 we will also give a generalization of this to arbitrary infinite fields.

\section{Framed MOdUli SPACES FOR QUIVER $A_{n-1}^{(1)}$}

In the three remaining sections we work over the arbitrary infinite field $\mathbb{k}$.

We will see that in attempts to apply Reineke's construction to quivers with oriented cycles we have to tackle Grassmannians in infinite dimensional spaces that do not carry a natural structure of algebraic variety. However, over an algebraically closed field the quotient $\operatorname{Rep}^{s}(Q, \alpha, \zeta) / / G L(\alpha)$ is projective over $\operatorname{Rep}(Q, \alpha, \zeta) / / G L(\alpha)$; see [9]. So, we can consider the natural projection

$$
\pi_{s}: \operatorname{Rep}^{s}(Q, \alpha, \zeta) / / G L(\alpha) \rightarrow \operatorname{Rep}(Q, \alpha, \zeta) / / G L(\alpha)
$$

and investigate its fibers. This also gives an idea of what to do when we do not possess a total framed moduli space. 
Let $Q$ be a cyclic quiver of type $A_{n-1}^{(1)}$ :

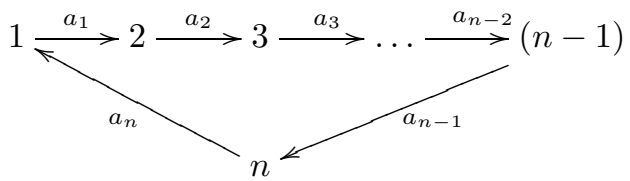

This notation for vertices and arrows of the quiver will be used throughout Sections 4 and 5 . There is a map $\pi: \operatorname{Rep}(Q, \alpha, \zeta) \rightarrow \mathbb{k}[x]_{\alpha_{1}} \times \ldots \times \mathbb{k}[x]_{\alpha_{n}}$, where $\mathbb{k}[x]_{r}$ is a space of polynomials of degree not higher than $r+1$, sending a representation to the tuple of characteristic polynomials of $\tau_{i}$. In this section our aim is to describe, for each $y \in \mathbb{k}[x]_{\alpha_{1}} \times \ldots \times \mathbb{k}[x]_{\alpha_{n}}$, the moduli space of representations belonging to $\pi^{-1}(y)$ for a cyclic quiver $Q$ of type $A_{n-1}^{(1)}$ :

To each path $\sigma$ in $Q$ we associate a linear function $\sigma^{*} \in(\mathbb{k} Q)^{*}$, taking values

$$
\sigma^{*}\left(\sigma^{\prime}\right)=\left\{\begin{array}{l}
1, \text { for } \sigma^{\prime}=\sigma \\
0, \text { otherwise }
\end{array}\right.
$$

for a path $\sigma^{\prime}$. Further, for $\sigma: i \rightsquigarrow j$ denote $V_{i}^{(\sigma)}:=\mathbb{k} \sigma^{*} \subseteq(\mathbb{k} Q)^{*}$ and set $\tau_{i}=a_{i-1} a_{i-2} \ldots a_{i+1} a_{i}$, the shortest path starting in $i$. Consider the $\mathbb{k} Q$-module $J$ with

$$
(J)_{i}:=\prod_{\sigma: i \rightsquigarrow k} V_{k}^{(\sigma)}=\prod_{j=0}^{\infty} V_{i}^{\left(\tau_{i}^{j}\right)} \times \prod_{j=0}^{\infty} V_{i+1}^{\left(\tau_{i+1}^{j} a_{i}\right)} \times \ldots \times \prod_{j=0}^{\infty} V_{i-1}^{\left(\tau_{i-1}^{j} a_{i-2} \ldots a_{i+1} a_{i}\right)} .
$$

Here we identify $\tau_{i}^{0}$ with $e_{i}$. Furthermore, we use the symbol of direct product instead of direct sum, since we allow our tuples to contain infinitely many nonzero terms. It should be explained how $a_{i} \in \mathbb{k} Q$ acts on $J$. Considering the restrictions of $a_{i}$ to the summands of (4) we have

$$
a_{i}: J_{i} \supseteq \prod_{j=0}^{\infty} V_{k}^{\left(\tau_{k}^{j} a_{k-1} \ldots a_{i+1} a_{i}\right)} \longrightarrow \prod_{j=0}^{\infty} V_{k}^{\left(\tau_{k}^{j} a_{k-1} \ldots a_{i+1}\right)} \subseteq J_{i+1},
$$

for $k \neq j$, where $a_{i}$ acts as the componentwise isomorphism $V_{k}^{\left(\tau_{k}^{j} a_{k-1} \ldots a_{i+1} a_{i}\right)} \stackrel{\mathrm{id}}{\longrightarrow}$ $V_{k}^{\left(\tau_{k} a_{k-1} \ldots a_{k+1}\right)}$. In cases where $k=i$ a shift $J_{i} \supseteq V_{i}^{\left(\tau_{i}^{j}\right)} \stackrel{\mathrm{id}}{\longrightarrow} V_{k}^{\left(\tau_{i}^{j-1} a_{i-1} \ldots a_{i+1}\right)} \subseteq J_{i+1}$ takes place and, in particular, $a_{i}: J_{i} \supseteq V_{i}^{\left(e_{i}\right)} \rightarrow 0$.

We will now try to make our notation more convenient. Since each $\prod_{j=0}^{\infty} V_{k}^{\left(\tau_{k}^{j} a_{k-1} \ldots a_{i+1} a_{i}\right)}$ is isomorphic as a vector space to $c_{\infty} \otimes V_{k}$, where $c_{\infty}$ is the space $\mathbb{k}^{\mathbb{Z} \geqslant 0}$, we may rewrite $J_{i}$ as $J_{i}=\left(c_{\infty} \otimes V_{1}\right) \oplus\left(c_{\infty} \otimes V_{2}\right) \oplus \ldots \oplus\left(c_{\infty} \otimes V_{n}\right)$, for $i=1, \ldots, n$. The $\mathbb{k} Q$-module structure will be translated as $a_{i} \cdot\left(G_{1}, \ldots, G_{n}\right)=$ $\left(G_{1}, \ldots, G_{i-1}, \delta G_{i}, G_{i+1}, \ldots, G_{n}\right)$ (elements of $c_{\infty} \otimes V_{i}$ can be regarded as vectors $G=\left(g_{1}, \ldots, g_{\zeta_{i}}\right), g_{j} \in c_{\infty}$; in this situation we define $\delta$ as a componentwise left shift).

For a pair $(M, f) \in \operatorname{Rep}(Q, \alpha, \zeta)$ define as before the map $\Phi_{(M, f)}=\left(\varphi_{i}\right)_{i=1}^{n}$ : $M \rightarrow J$ by $\varphi_{i}(m)=\left(f_{j}(\tau m)\right)_{\tau: i \rightsquigarrow j}$. Here we mean that the component of $\varphi(m)$ that corresponds to $V_{k}^{(\tau)} \subseteq J_{i}$ equals $f(\tau m)$. According to the above decomposition we get

$$
\varphi_{i}(m)=\left(f_{i}\left(\mathfrak{e x p}\left(\tau_{i}\right) m\right), f_{i+1}\left(\mathfrak{x} \mathfrak{x} \mathfrak{p}\left(\tau_{i+1}\right) a_{i} m\right), \ldots, f_{i-1}\left(\mathfrak{e x p}\left(\tau_{i-1}\right) a_{i-2} \ldots a_{i+1} a_{i} m\right)\right),
$$


where $\mathfrak{e x p}\left(\tau_{r}\right): V_{p} \rightarrow c_{\infty} \otimes V_{r}$ are linear operators defined by $v \mapsto\left(v, \tau_{r} v, \tau_{r}^{2} v, \ldots\right.$, $\left.\tau_{p}^{s} r v, \ldots\right)$ and $f_{r}$ act as componentwise multiplications.

Now it will be shown that the above constructed map shares some of the useful properties of its finite dimensional analog.

Lemma 4.1. (i) The map $\Phi_{(M, f)}$ is a $\mathbb{k} Q$-homomorphism.

(ii) The subspace $\operatorname{ker} \Phi_{(M, f)}$ is the maximal $\mathbb{k} Q$-submodule of $M$ contained in ker $f$.

(iii) A pair $(M, f)$ is stable if and only if $\Phi_{(M, f)}$ is injective.

Proof. (i) Let $a_{i}$ be an arrow in $Q$. Denote by $\varphi_{i}$ the components of $\Phi_{(M, f)}$. It is sufficient to show that $\left.\left(a_{i}\right)\right|_{J_{i}} \circ \phi_{i}=\left.\phi_{i+1} \circ\left(a_{i}\right)\right|_{M_{i}}$.

Observe that, for all $r$, we have $\delta\left(f_{r} \mathfrak{e x p}\left(\tau_{r}\right)\right)=f_{r} \mathfrak{e x p}\left(\tau_{r}\right) \tau_{r}$. Hence,

$$
\begin{aligned}
a_{i} & \cdot \varphi_{i}(m) \\
& =\left(\delta f_{i}\left(\mathfrak{e x p}\left(\tau_{i} t\right) m\right), f_{i+1}\left(\mathfrak{e x p}\left(\tau_{i+1} t\right) a_{i} m\right), \ldots, f_{i-1}\left(\mathfrak{e x p}\left(\tau_{i-1} t\right) a_{i-2} \ldots a_{i+1} a_{i} m\right)\right) \\
& =\left(f_{i}\left(\mathfrak{e x p}\left(\tau_{i} t\right) \tau_{i} m\right), f_{i+1}\left(\mathfrak{e x p}\left(\tau_{i+1} t\right) a_{i} m\right), \ldots, f_{i-1}\left(\mathfrak{e x p}\left(\tau_{i-1} t\right) a_{i-2} \ldots a_{i+1} a_{i} m\right)\right) \\
& =\left(f_{i}\left(\mathfrak{e} \mathfrak{x} \mathfrak{p}\left(\tau_{i} t\right) a_{i-1} \ldots a_{i+1} \cdot a_{i} m\right), f_{i+1}\left(\mathfrak{e x p}\left(\tau_{i+1} t\right) \cdot a_{i} m\right), \ldots,\right. \\
& =\phi_{i+1}\left(a_{i} m\right) .
\end{aligned}
$$

Thus, $\Phi_{(M, f)}$ is a $\mathbb{k} Q$-homomorphism.

(ii) It follows from (i) that $\operatorname{ker} \Phi_{(M, f)}$ is a $\mathbb{k} Q$-submodule of $M$. Let $N \subseteq M$ be a submodule contained in ker $f$. Then for a path $\tau$ we have $\tau(N) \subseteq N$ and, therefore, $\Phi_{(M, f)}(N)=0$. Consequently, $N \subseteq \operatorname{ker} \Phi_{(M, f)}$.

(iii) It is a direct consequence of (ii).

In the finite dimensional case, to each element $(M, f) \in \operatorname{Rep}^{s}(Q, \alpha, \zeta)$ there is associated a submodule of $J$ with dimension vector $\alpha$ that is a point in the Grassmannian of submodules $\operatorname{Gr}_{\alpha}^{\mathbb{k} Q}(J)$. Our next aim is to prove that the same holds in the current setting.

Let $U \subseteq J$ be a $\mathbb{k} Q$-submodule with dimension vector $\alpha$. For each $i=1, \ldots, n$ we have an inclusion $a_{i}\left(U_{i}\right) \subseteq U_{i+1}$. This implies that $\tau_{i}\left(U_{i}\right) \subseteq U_{i}$. We know that $\tau_{i}: J_{i}=\bigoplus_{j=1}^{n}\left(c_{\infty} \otimes V_{j}\right) \rightarrow \bigoplus_{j=1}^{n}\left(c_{\infty} \otimes V_{j}\right)=J_{i+1},\left(G_{1}, \ldots, G_{n}\right) \mapsto\left(\delta G_{1}, \ldots, \delta G_{n}\right)$. Hence if $U$ is a submodule, then it is preserved by componentwise left shifts. This means that $U_{i}$ are $\delta$-invariant subspaces in $J_{i}$ (note that $\bigoplus_{j=1}^{n}\left(c_{\infty} \otimes V_{j}\right) \cong c_{\infty} \otimes$ $\left(\bigoplus_{j=1}^{n} V_{j}\right)$, and so its elements may be considered as $\sum_{j=1}^{n} \zeta_{j}$-tuples of sequences). Further, let $D^{(i)}=\left(d_{p q}^{(i)}\right)$ be the matrix of the restricted operator $\left.\delta\right|_{U_{i}}$ and set $k=\alpha_{i}, m=\sum_{j=1}^{n} \zeta_{j}$. Fix a base $\bar{g}_{1}, \ldots, \bar{g}_{k}$ in $U_{i}$ (here we consider $\bar{g}_{j}$ as an $m$-tuple of sequences: $\left.\bar{g}_{j}=\left(g_{j l}\right)_{l=1}^{n}\right)$. Then, for all $j$, we get

$$
\delta \bar{g}_{j}=d_{1 j}^{(i)} \bar{g}_{1}+d_{2 j}^{(i)} \bar{g}_{2}+\ldots+d_{k j}^{(i)} \bar{g}_{k} .
$$

For a vector sequence $\bar{h}$ denote $\bar{h}(0):=(\bar{h})_{0}$. It is an easy calculation to check that

$$
\left(\bar{g}_{1}, \ldots, \bar{g}_{k}\right)=\left(\bar{g}_{1}(0), \ldots, \bar{g}_{k}(0)\right) \mathfrak{e x p}\left(D^{(i)}\right) .
$$

Now Cayley-Hamilton's Theorem implies that $\chi_{i}\left(D^{(i)}\right)=0$ in $U_{i}$, where $\chi_{i}$ is a characteristic polynomial of $D^{(i)}$. Thus each vector sequence $\bar{g} \in U_{i}$ satisfies the equation $\chi_{i}(\delta) \bar{g}=0$. 
We can finally show that the above constructed correspondence between stable pairs and points in Grassmannian is one-to-one.

Proposition 4.2. The map $\Phi: \operatorname{Rep}^{s}(Q, \alpha, \zeta) \rightarrow \operatorname{IHom}_{\alpha}^{\mathbb{k} Q}(J),(M, f) \mapsto \Phi_{(M, f)}$ is a bijection.

Proof. We need to show that by having an inclusion $\Phi \in \operatorname{IHom}_{\alpha}^{\mathbb{k} Q}(J)$ we can uniquely recover a pair $(M, f)$. First of all note that since for each $j, r$ we have $\tau_{j}^{r} a_{j-1} \ldots a_{i}=a_{j-1} \ldots a_{i} \tau_{i}^{r}$, the map $\varphi_{i}$ may be written as

$$
\begin{gathered}
\varphi_{i}(m)=\left(f_{i}\left(\mathfrak{x} \mathfrak{x} \mathfrak{p}\left(\tau_{i} t\right) m\right), f_{i+1}\left(a_{i} \mathfrak{e x p}\left(\tau_{i} t\right) m\right), \ldots,\right. \\
\left.f_{i-1}\left(a_{i-2} \ldots a_{i+1} a_{i} \mathfrak{e} \mathfrak{k} \mathfrak{p}\left(\tau_{i} t\right) m\right)\right) .
\end{gathered}
$$

Now let $U=\left(U_{i}\right)_{i=1}^{n} \subseteq J$ be a submodule with dimension vector $\alpha$. Also let $U_{i}=\operatorname{span}\left\{\bar{g}_{1}^{(i)}, \ldots, \bar{g}_{\alpha_{i}}^{(i)}\right\}$.

Now recall equality (5), setting $\bar{g}_{r}=\bar{g}_{r}^{(i)}$. The matrices $\left(\bar{g}_{1}^{(i)}, \ldots, \bar{g}_{\alpha_{i}}^{(i)}\right)$ and $\left(\bar{g}_{1}^{(i)}(0), \ldots, \bar{g}_{\alpha_{i}}^{(i)}(0)\right)$ may be divided into horizontal blocks of size $\zeta_{j} \times \alpha_{i}$, those blocks corresponding to the natural projections $P_{j}: J_{i} \rightarrow c_{\infty} \otimes V_{j}$. Thus, for each $j$, we obtain

$$
\left(P_{j} \bar{g}_{1}^{(i)}, \ldots, P_{j} \bar{g}_{\alpha_{i}}^{(i)}\right)=\left(P_{j} \bar{g}_{1}^{(i)}(0), \ldots, P_{j} \bar{g}_{\alpha_{i}}^{(i)}(0)\right) \mathfrak{e x p} D^{(i)} t \cdot E_{\alpha_{i}},
$$

where $E_{\alpha_{i}}$ is the identity matrix of size $\alpha_{i} \times \alpha_{i}$. These equalities can be interpreted as follows: there is a map $\Psi=\left(\Psi_{i j}\right)_{i, j=1}^{n}$ with

$$
\Psi_{i j}=\left(P_{j} \bar{g}_{1}^{(i)}(0), \ldots, P_{j} \bar{g}_{\alpha_{i}}^{(i)}(0)\right) \mathfrak{e x p} D^{(i)} t: M_{i} \rightarrow c_{\infty} \otimes V_{j} \subseteq J_{i},
$$

where $M$ is a graded vector space with dimension vector $\alpha$; one may easily establish that $\Psi$ is bijective. We need to show that $\Psi=\Phi_{(M, f)}$ for a map $f: M \rightarrow V$ and a certain $\mathbb{k} Q$-module structure on $M$.

As for the module structure, it is quite clear: viewing (6) as formulas defining the natural inclusion $U \hookrightarrow J$, we set $M=U$. Further, $f=\left(f_{i}\right)_{i=1}^{n}$ is defined as a tuple of compositions $f_{i}: M_{i} \hookrightarrow J_{i} \rightarrow V_{i}$, where $V_{i}$ should be regarded as the component of $J_{i}$ associated to $e_{i}$ (we mean $V_{i}^{\left(e_{i}\right)} \subseteq V_{i}^{\left(e_{i}\right)} \oplus \bigoplus_{j=1}^{\infty} V_{i}^{\left(\tau_{i}^{j}\right)}=\left(c_{\infty} \otimes V_{i}\right)$ ). Hence,

$$
f_{i}=\left.\Psi_{i i}\right|_{t=0}: m \mapsto\left(P_{i} \bar{g}_{1}^{(i)}(0), \ldots, P_{i} \bar{g}_{\alpha_{i}}^{(i)}(0)\right) m .
$$

Now it is left to prove that $(7)$ defines a map $\Psi$ coinciding with $\Phi_{(M, f)}$ for the above $M$ and $f$. From (6) it follows that it is sufficient to show that

$$
\left(P_{j} \bar{g}_{1}^{(i)}(0), \ldots, P_{j} \bar{g}_{\alpha_{i}}^{(i)}(0)\right) \mathfrak{e x p} D^{(i)} t=f_{j} \cdot a_{j-1} \ldots a_{i} \mathfrak{e x p}\left(\tau_{i} t\right),
$$

for $j \neq i$. But $D^{(i)}$ is just a notation for the matrix of $\tau_{i}$, so $\mathfrak{e x p}\left(D^{(i)} t\right) \equiv \mathfrak{e x p}\left(\tau_{i} t\right)$. Canceling this exponent, we turn (8) into

$$
\left(P_{j} \bar{g}_{1}^{(i)}(0), \ldots, P_{j} \bar{g}_{\alpha_{i}}^{(i)}(0)\right)=\left(P_{j} \bar{g}_{1}^{(j)}(0), \ldots, P_{j} \bar{g}_{\alpha_{j}}^{(j)}(0)\right) \cdot a_{j-1} \ldots a_{i} .
$$

We are going to prove this as a matrix equality, and so we can immediately write

$$
\left(a_{j-1} \ldots a_{i} \bar{g}_{1}^{(i)}, \ldots, a_{j-1} \ldots a_{i} \bar{g}_{\alpha_{i}}^{(i)}\right)=\left(\bar{g}_{1}^{(j)}, \ldots, \bar{g}_{\alpha_{j}}^{(j)}\right) \cdot a_{j-1} \ldots a_{i} .
$$


Descending to the level of projections, we obtain

$$
\left(P_{j} a_{j-1} \ldots a_{i} \bar{g}_{1}^{(i)}, \ldots, P_{j} a_{j-1} \ldots a_{i} \bar{g}_{\alpha_{i}}^{(i)}\right)=\left(P_{j} \bar{g}_{1}^{(j)}, \ldots, P_{j} \bar{g}_{\alpha_{j}}^{(j)}\right) \cdot a_{j-1} \ldots a_{i},
$$

but $a_{r}$ acts on $J_{i}$ as (id, $\ldots, \delta, \ldots$, id), where the shift takes place only at the $r$-th position. Consequently, the product $a_{j-1} \ldots a_{i}$ does not change the $j$-th projection of $\bar{g}^{(i)}$, implying that

$$
\left(P_{j} a_{j-1} \ldots a_{i} \bar{g}_{1}^{(i)}, \ldots, P_{j} a_{j-1} \ldots a_{i} \bar{g}_{\alpha_{i}}^{(i)}\right)=\left(P_{j} \bar{g}_{1}^{(i)}, \ldots, P_{j} \bar{g}_{\alpha_{i}}^{(i)}\right),
$$

and the required equality follows. The proposition is proved.

This proposition cannot be used to describe (as was done in Section 3) the total framed moduli space. So, we restrict our attention to fibers of $\pi$.

Lemma 4.3. Let $\bar{\chi}=\left(\chi_{1}, \ldots, \chi_{n}\right)$ be an admissible tuple of polynomials and $h_{0}=$ $x, h_{1}, \ldots, h_{N}$ be all different irreducible factors of $\chi_{1}, \ldots, \chi_{n}$ in $\mathbb{k}[x]$. Also let $r_{i j}$ be the multiplicity of $h_{j}$ as a factor of $\chi_{i}$. If $j \neq 0$, then $r_{1 j}=\ldots=r_{n j}$.

Proof. Let $M \in \pi^{-1}(\bar{\chi})$. Consider the block decomposition of $M_{i}$ with respect to $\tau_{i}$. Then $r_{i j} \cdot \operatorname{deg}\left(h_{j}\right)$ is the dimension of the subspace $M_{i}^{h_{j}}:=\left\{m \in M_{i} \mid h_{j}^{r_{i j}} m=0\right\}$. If $h_{j}(x) \neq x$, the restriction of $\tau_{i}=a_{i-1} \ldots a_{i}$ on $M_{i}^{h_{j}}$ is nondegenerate. Therefore, $a_{p-1} \ldots a_{i}\left(M_{i}^{h_{j}}\right) \cap \operatorname{ker} a_{p}=0$, for all $p=1, \ldots, n$. As $\tau_{p} a_{p-1} \ldots a_{i}=a_{p-1} \ldots a_{i} \tau_{i}$, all $\left.\tau_{p}\right|_{a_{p-1} \ldots a_{i}\left(M_{i}^{h_{j}}\right)}$ are conjugate and therefore have the same characteristic polynomials.

Fix a tuple $\bar{\chi}$. Let $h_{j}$ and $r_{i j}$ be as in Lemma 4.3. Define

$$
\underline{r}_{j}=\left(r_{1 j} \operatorname{deg}\left(h_{j}\right), \ldots, r_{n j} \operatorname{deg}\left(h_{j}\right)\right),
$$

for $j=0, \ldots, N$. Consider the submodules $J\left(h_{j}, \underline{r}_{j}\right)$ of $J$, where $J\left(h_{j}, \underline{r}_{j}\right)_{i}$ is the

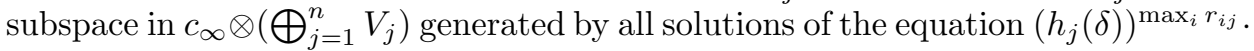
$\bar{g}=0$. It is not hard to see that these submodules are finite dimensional.

Lemma 4.4. For $M \in \pi^{-1}(\bar{\chi})$, the image $\Phi_{(M, f)}(M)$ lies in $\bigoplus_{j=0}^{N} J\left(h_{j}, \underline{r}_{j}\right)$.

Proof. As was shown before, each vector function in $\left(\Phi_{(M, f)}(M)\right)_{i}$ satisfies the equation $\chi_{i}(\delta) \cdot \bar{g}=0$, where $\chi_{i}$ is a characteristic polynomial of $\tau_{i}$ as an operator in $M_{i}$. If $\chi_{i}(x)=h_{0}(x)^{r_{i 0}} h_{1}(x)^{r_{i 1}} \ldots h_{N}(x)^{r_{i N}}$, then clearly $\left(\Phi_{(M, f)}(M)\right)_{i} \subseteq$ $J\left(h_{0}, \underline{r}_{0}\right)_{i} \oplus J\left(h_{1}, \underline{r}_{1}\right)_{i} \oplus \ldots \oplus J\left(\lambda_{N}, \underline{r}_{N}\right)_{i}$. This yields the required inclusion.

Now let $W=\bigoplus_{j=0}^{N} J\left(h_{j}, \underline{r}_{j}\right)$ and $\Phi_{(M, f)}^{j}$ be $\Phi_{(M, f)}$, followed by the projection on $J\left(h_{j}, \underline{r}_{j}\right)$ along $\bigoplus_{p \neq j} J\left(h_{p}, \underline{r}_{p}\right)$.

Lemma 4.5. A stable pair $(M, f)$ is in $\pi_{G L(\alpha)}^{-1}(\bar{\chi})$ if and only if the dimension vector of each $\Phi_{(M, f)}^{j}(M) \subseteq J\left(\lambda_{j}, \underline{r}_{j}\right), j=0, \ldots, N$, equals $\underline{r}_{i j}$.

Proof. The "only if" part is straightforward. Conversely, if $(M, f)$ is a stable pair, we have $\Phi_{(M, f)}^{j}(M)=\bigoplus_{j=0}^{N} W(j)$, where $W(j)=W \cap J\left(h_{j}, \underline{r}_{j}\right)$, so that $W(j)_{i}=$ $W_{i}^{h_{j}}$. Since $\Phi_{(M, f)}$ is a $\mathbb{k} Q$-homomorphism, $\operatorname{dim} M_{i}^{h_{j}}=\operatorname{dim} W_{i}^{h_{j}}=r_{i j}$. This implies that the characteristic polynomial of $\tau_{i}$ acting on $M_{i}$ is $\prod_{j=0}^{N} h_{j}(x)^{r_{i j}}=$ $\chi_{i}(x)$. So, $(M, f) \in \pi_{G L(\alpha)}^{-1}(\bar{\chi})$. 
This lemma ensures that $\Phi: \operatorname{Rep}^{s}(Q, \alpha, \zeta) \rightarrow \operatorname{IHom}_{\alpha}^{\mathbb{k} Q}(J),(M, f) \mapsto \Phi_{(M, f)}$ restricted to $\pi^{-1}(\bar{\chi})$ induces a bijection

$$
\operatorname{Rep}^{s}(Q, \alpha, \zeta) \cap \pi^{-1}(\bar{\chi}) \rightarrow \prod_{j=0}^{N} \operatorname{IHom}_{\underline{r}_{j}}^{\mathbb{k} Q}\left(J\left(h_{j}, \underline{r}_{j}\right)\right) .
$$

As $J\left(h_{j}, \underline{r}_{j}\right)$ are finite dimensional, we can use Lemma 3.6 to prove that $\pi^{-1}(\bar{\chi}) / /$ $G L(\alpha)$ is isomorphic (this time as an algebraic variety) to $\prod_{j=0}^{N} \operatorname{Gr}_{\underline{r}_{j}}^{\mathbb{k} Q}\left(J\left(h_{j}, \underline{r}_{j}\right)\right)$.

The last ingredient we need is

Lemma 4.6. For $j>0$ we have $\operatorname{Gr}_{\underline{r}_{j}}^{\mathbb{k} Q}\left(J\left(h_{j}, \underline{r}_{j}\right)\right) \cong \operatorname{Gr}_{r_{1 j} \operatorname{deg}\left(h_{j}\right)}^{\mathbb{k} A_{0}^{(1)}}\left(J\left(h_{j}, r_{1 j} \operatorname{deg}\left(h_{j}\right)\right)\right)$.

Proof. As a vector space we may identify $J\left(h_{j}, r_{1 j} \operatorname{deg}\left(h_{j}\right)\right)$ with $J\left(h_{j}, \underline{r}_{j}\right)_{1}$. Moreover, the $\mathbb{k} Q$-module structure on $J\left(h_{j}, r_{1 j}\right)$ induces a $\mathbb{k} A_{0}^{(1)}$-module structure on $J\left(h_{j}, \underline{r}_{j}\right)_{1}$. Namely, the loop acts as $\tau_{1}$. There is an obvious morphism $\operatorname{Gr}_{\underline{r}_{j}}^{\mathbb{k} Q}\left(J\left(h_{j}, \underline{r}_{j}\right)\right)$ $\rightarrow \operatorname{Gr}_{r_{1 j} \operatorname{deg}\left(h_{j}\right)}^{\mathbb{k} A_{0}^{(1)}}\left(J\left(h_{j}, r_{1 j} \operatorname{deg}\left(h_{j}\right)\right)\right),\left(U_{i}\right)_{i=1}^{n} \mapsto U_{1}$. Its inverse may be defined as

$$
U \mapsto\left(a_{i-1} \ldots a_{1}(U)\right)_{i=1}^{n} .
$$

Collecting the results obtained, we can state the following.

Theorem 4.7. (1) Points of the quotient $\mathcal{M}^{s}(Q, \alpha, \zeta)$ are in one-to-one correspondence with points of the Grassmannian of submodules $\operatorname{Gr}_{\alpha}^{\mathbb{k} Q}(J)$.

(2) Let $\bar{\chi}=\left(\chi_{1}, \ldots, \chi_{n}\right)$ be an admissible tuple of polynomials, $h_{0}=x, h_{1}, \ldots, h_{N}$ be all different irreducible factors of $\chi_{1}, \ldots, \chi_{n}$, and $r_{i j}$ be the multiplicity of $h_{j}$ as a factor of $\chi_{i}$. Then

$$
\pi^{-1}(\bar{\chi}) / / G L(\alpha) \cong \operatorname{Gr}_{\underline{r}_{0}}^{\mathbb{k} Q}\left(J\left(h_{0}, \underline{r}_{0}\right)\right) \times \prod_{j=1}^{N} \operatorname{Gr}_{r_{1 j} \operatorname{deg}\left(h_{j}\right)}^{\mathbb{k} A_{(1)}^{(1)}}\left(J\left(h_{j}, r_{1 j} \operatorname{deg}\left(h_{j}\right)\right)\right),
$$

where $\underline{r}_{0}=\left(r_{10}, \ldots, r_{n 0}\right)$.

If $\mathbb{k}$ is one of the fields $\mathbb{C}$ or $\mathbb{R}$, the modules $J\left(h_{j}, \underline{r}_{j}\right)$ allow the following convenient interpretation. Consider the space $J^{!}=\bigoplus_{i=1}^{n} J_{i}^{!}$with $J_{i}^{!}=\left(\mathbb{k}[[t]] \otimes V_{1}\right) \oplus$ $\left(\mathbb{k}[[t]] \otimes V_{2}\right) \oplus \ldots \oplus\left(\mathbb{k}[[t]] \otimes V_{n}\right)$, for $i=1, \ldots, n$, with the following $\mathbb{k} Q$-module structure: $a_{i} \cdot\left(G_{1}, \ldots, G_{n}\right)=\left(G_{1}, \ldots, G_{i-1}, \frac{d}{d t} G_{i}, G_{i+1}, \ldots, G_{n}\right)$ (elements of $\mathbb{k}[[t]] \otimes V_{i}$ can be regarded as vectors $G=\left(g_{1}, \ldots, g_{\zeta_{i}}\right), g_{j} \in \mathbb{k}[[t]]$; in this situation $\frac{d}{d t}$ is a componentwise formal differentiation). Further, define a map $T=\left(T_{i}\right)_{i=1}^{n}: J \rightarrow J^{!}$ by

$$
\begin{gathered}
T_{i}: J_{i}=\left(c_{\infty} \otimes V_{i}\right) \oplus\left(c_{\infty} \otimes V_{i+1}\right) \oplus\left(c_{\infty} \otimes V_{i+2}\right) \oplus \ldots \oplus\left(c_{\infty} \otimes V_{i-1}\right) \\
\longrightarrow J_{i}^{!}=\left(\mathbb{k}[[t]] \otimes V_{i}\right) \oplus\left(\mathbb{k}[[t]] \otimes V_{i+1}\right) \oplus\left(\mathbb{k}[[t]] \otimes V_{i+2}\right) \oplus \ldots \oplus\left(\mathbb{k}[[t]] \otimes V_{i-1}\right) .
\end{gathered}
$$

This map is componentwise, and its components $T_{i k}:\left(c_{\infty} \otimes V_{k}\right) \rightarrow\left(\mathbb{k}[[t]] \otimes V_{k}\right)$ are

$$
T_{i j}\left(\bar{c}_{0}, \bar{c}_{1}, \ldots, \bar{c}_{k}, \ldots\right)=\sum_{k=0}^{\infty} \frac{1}{k !} t^{k} \bar{c}_{k} .
$$

It is easy to check that this map is a $\mathbb{k} Q$-isomorphism. 
Furthermore, we consider the map $\Phi_{(M, f)}^{!}=\left(\varphi_{i}^{!}\right)_{i=1}^{n}:=T \circ \Phi_{M, f}$. It is not hard to see that

$$
\begin{gathered}
\varphi_{i}^{!}(m)=\left(f_{i}\left(\exp \left(\tau_{i} t\right) m\right), f_{i+1}\left(\exp \left(\tau_{i+1} t\right) a_{i} m\right), f_{i+2}\left(\exp \left(\tau_{i+2} t\right) a_{i+1} a_{i} m\right), \ldots,\right. \\
\left.f_{i-1}\left(\exp \left(\tau_{i-1} t\right) a_{i-2} \ldots a_{i+1} a_{i} m\right)\right) .
\end{gathered}
$$

This map obviously enjoys the properties of its precursor. Now denote by $\mathcal{A}[t]$ the subspace in $\mathbb{k}[[t]]$ consisting of power series converging for all $t$. For $\mathbb{k}=\mathbb{C}$, this conditions means that the series is a Taylor series of a holomorphic function, and hence the uniqueness theorem implies that $\mathcal{A}[t]$ is the ring of entire functions $\mathcal{O}(\mathbb{C})$. For $\mathbb{k}=\mathbb{R}$ it may also be viewed as a space of functions that coincide with their Taylor series in 0 in any point. So, we replace $J^{!}$by $J^{! !}$with $J_{i}^{! !}=$ $\left(\mathcal{A}[t] \otimes V_{i}\right) \oplus\left(\mathcal{A}[t] \otimes V_{i+1}\right) \oplus\left(\mathcal{A}[t] \otimes V_{i+2}\right) \oplus \ldots \oplus\left(\mathcal{A}[t] \otimes V_{i-1}\right)$ and the same action of $\mathbb{k} Q$. It is again possible to prove that there is a bijection between $\mathcal{M}(Q, \alpha, \zeta)$ and $\operatorname{Gr}_{\alpha}^{\mathbb{k} Q}\left(J^{! !}\right)$. Moreover, $J\left(h_{j}, \underline{r}_{j}\right)_{i}$ is now the subspace in $\mathcal{A}[t] \otimes\left(\bigoplus_{j=1}^{n} V_{j}\right)$ generated by all solutions of the differential equation $h_{j}\left(\frac{d}{d t}\right)^{\max _{i} r_{i j}} \cdot \bar{g}=0$. For $\mathbb{k}=\mathbb{C}$ all the possible $h_{j}(x)$ are of the form $\left(x-\lambda_{j}\right)$, for some $\lambda_{j} \in \mathbb{C}$, and for $\mathbb{k}=\mathbb{R}$ polynomials of degree 2 with negative discriminant may also occur. In both cases one can easily write a basis in $J\left(h_{j}, \underline{r}_{j}\right)_{i}$.

\section{An EXPLiCIT REALIZATION OF FIBERS}

In this section the ground field $\mathbb{k}$ is either $\mathbb{C}$ or $\mathbb{R}$. This allows us to use the language of functions introduced at the end of Section 4.

Let $Q$ be a Jordan quiver consisting of a single vertex and a single loop (both the loop and the corresponding operator in a representation will be denoted by $a$ ). Also set $\alpha=(m), \zeta=(q)$. This is the case when our construction becomes as clear as possible.

It is evident that the standard categorical quotient for the action $G L(m)$ : $\operatorname{Rep}(Q, m)$ is isomorphic to $\mathbb{A}^{m}$ : points of the quotient are tuples of characteristic polynomial coefficients of the operator corresponding to the arrow $a$. Having this in mind we will further assume that $\mathcal{M}(Q, m)$ is embedded in $\mathbb{k}[t]_{m}$.

We have $J^{! !}=J_{1}^{! !}=\mathcal{A} \otimes V_{1}$, and the map $\Phi_{(M, f)}^{!}$becomes $\varphi(m)=f_{1}(\exp (a t) m)$. Further, for a subspace $U \in \operatorname{Gr}_{m}\left(J^{! !}\right)$the equivalence $U \in \operatorname{Im}\left(\Phi^{!}\right) \Leftrightarrow \frac{d}{d t}(U) \subseteq U$ holds. Thus, the fiber over $\chi \in \mathbb{k}[x]$ is precisely $\operatorname{Gr}_{m}^{\frac{d}{d t}}\left(J_{\chi}^{! !}\right)$(the Grassmannian of $m$-dimensional $\frac{d}{d t}$-invariant subspaces in $J_{\chi}^{! !}$).

Imagine $q=1$. We then have ordinary functions instead of vector ones, and the dimension of the solution space of the differential equation $\chi\left(\frac{d}{d t}\right) g=0$ equals $m$. So each Grassmannian $\operatorname{Gr}^{\frac{d}{d t}}\left(J_{\chi}^{! !}\right)$consists in this case of a single subspace. In other words, in each fiber of the projection $\pi_{s}: \mathcal{M}^{s}(Q, m, 1) \rightarrow \mathcal{M}(Q, m, 1)$ there is at most one point. Such a result is rather upsetting, though the situation will be more favorable for $q>1$. We may even guarantee that for $q=m$ each $\mathbb{k} Q$-module $M$ arises as a member of a stable pair $(M, f) \in \operatorname{Rep}^{s}(Q, m, q)$ (it is, for instance, $(M$, id $))$.

Proposition 5.1. For all $\lambda \in \mathbb{k}$ we have $\operatorname{Gr}_{m}^{\frac{d}{d t}}(J(x-\lambda, m)) \cong \operatorname{Gr}_{m}^{\frac{d}{d t}}(J(x, m))$.

Proof. For $\mathbb{k}=\mathbb{C}$ it may be deduced from [6, Theorem 4.1]. However, this simple statement is true over an arbitrary field. Indeed, both in $J(x-\lambda, m)$ and in $J(x, m)$ the operator $\frac{d}{d t}$ can be brought to the Jordan normal form. Moreover, 
we may arrange the bases in such a way that these normal forms will only differ by diagonal coefficients. Now, consider a linear map $F$ sending basic vectors of $J(x-\lambda, m)$ to the corresponding ones in $J(x, m)$. Clearly $F$ is an isomorphism of linear spaces, and both it and its inverse send $\frac{d}{d t}$-stable subspaces to $\frac{d}{d t}$-stable subspaces.

Notice that for $\mathbb{k}=\mathbb{C}$ this together with Theorem 4.7 gives the first statement of [6. Theorem 4.1] for cyclic quivers of type $A_{n}^{(1)}$, i.e. the presentation recalled at the end of Section 3.

Proposition 5.2. If $\mathbb{k}=\mathbb{R}$ and $h_{1}, h_{2}$ are irreducible polynomials of degree 2 , then $\operatorname{Gr}_{m}^{\frac{d}{d t}}\left(J\left(h_{1}, m\right)\right) \cong \operatorname{Gr}_{m}^{\frac{d}{d t}}\left(J\left(h_{2}, m\right)\right)$ for all even $m$.

Proof. Again we can use the fact that in both $J\left(h_{1}, m\right)$ and $J\left(h_{2}, m\right)$ the operator $\frac{d}{d t}$ can be brought to an analog of Jordan normal form and establish an isomorphism that preserves $\frac{d}{d t}$-stable subspaces.

By Theorem 4.7, to describe the fibers of $\pi_{s}$ it is sufficient to tackle with Grassmannians $\operatorname{Gr}_{r \operatorname{deg}(h)}^{\frac{d}{d t}}(J(h, r \operatorname{deg}(h)))$, where $h$ is irreducible over $\mathbb{k}$.

Theorem 5.3. Let $m, q$ be positive integers.

(a) If $m=1$ and $\lambda \in \mathbb{k}$, then $\operatorname{Gr}_{m}^{\frac{d}{d t}}(J(x-\lambda, m)) \cong \mathbb{P}^{q-1}$.

(b) If $m>1$ and $\lambda \in \mathbb{k}$, then $\operatorname{Gr}_{m}^{\frac{d}{d t}}(J(x-\lambda, m))$ is isomorphic to the subvariety in $\mathbb{P}\left(\bigwedge^{m} \mathbb{k}^{q m}\right)$ given by the following equations:

(i) The Plücker relations.

(ii) For each tuple $0 \leqslant i_{1}<\ldots<i_{m}$ :

$$
\sum_{\substack{\epsilon_{1}, \ldots, \epsilon_{m} \in\{0 ; 1\}^{m} \\ \epsilon_{1}^{2}+\ldots+\epsilon_{m}^{2} \neq 0}} p_{i_{1}+q \epsilon_{1}, \ldots, i_{m}+q \epsilon_{m}}=0 .
$$

(c) For $\mathbb{k}=\mathbb{R}$ and $h$ an irreducible degree 2 polynomial we number coordinates in $\mathbb{k}^{2 q m}$ by residue classes modulo $2 m q$. Then $\operatorname{Gr}_{2 m}^{\frac{d}{d t}}(J(h, 2 m))$ is isomorphic to the subvariety in $\mathbb{P}\left(\bigwedge^{2 m} \mathbb{k}^{2 q m}\right)$ given by the following equations:

(i) The Plücker relations.

(ii) For each tuple $0 \leqslant i_{1}<\ldots<i_{2 m}$ :

$$
\begin{aligned}
& p_{q l_{1}+\nu_{1}, \ldots, q l_{2 m}+\nu_{m}} \\
& =\sum_{(\epsilon, \delta) \in \Delta}(-1)^{\sum_{i: \delta_{i} \neq 0} \theta\left(l_{i}\right)} p_{q\left(l_{1}+\epsilon_{1}+m \delta_{1}\right)+\nu_{1}, \ldots, q\left(l_{2 m}+\epsilon_{2 m}+m \delta_{2 m}\right)+\nu_{m}}, \\
& \text { where }
\end{aligned}
$$

$$
\theta(l)= \begin{cases}1, & \text { if } m \leqslant l<2 m, \\ 0, & \text { otherwise, }\end{cases}
$$

and $\Delta$ is the set of the pair of tuples

$$
\left(\left(\epsilon_{1}, \ldots, \epsilon_{2 m}\right),\left(\delta_{1}, \ldots, \delta_{2 m}\right)\right) \in\{0 ; 1\}^{2 m} \times\{0 ; 1\}^{2 m}
$$

satisfying conditions $\sum_{i} \epsilon_{i}^{2}+\sum_{i} \delta_{i}^{2} \neq 0$ and $\epsilon_{i}+\delta_{i}=1$, for all $i$. 
Proof. (a) For $m=1$, we have $\chi(x)=(x-\lambda)$, where $\lambda \in \mathbb{k}$. But each onedimensional $\frac{d}{d t}$-invariant subspace in $J(x-\lambda, 1)$ is generated by a vector function $\bar{g}$ satisfying $\frac{d}{d t} \bar{g}=\lambda \bar{g}$; that is, by $\left(\alpha_{1} e^{\lambda t}, \ldots, \alpha_{q} e^{\lambda t}\right)$, where $\alpha_{i} \in \mathbb{k}$. Hence, $\operatorname{Gr}_{1}^{\frac{d}{d t}}(J(x-\lambda, 1))$ is a projectivization of the linear span of such functions, i.e. it is isomorphic to $\mathbb{P}^{q-1}$.

(b) From Proposition 5.1 it follows that $\pi^{-1}(\chi)$ is in this case isomorphic to $\operatorname{Gr}_{m}^{\frac{d}{d t}}(J(x-1, m))$. So, we will be working only with the latter Grassmannian.

It is necessary to fix some notation. For $i=1, \ldots, q, j=0, \ldots, m-1$, set $e_{i j}=\left(0, \ldots, 0, \frac{1}{j !} t^{j} e^{t}, 0, \ldots, 0\right)$, where the only nonzero component is the $i$-th one. Now let $U \in \operatorname{Gr}_{m}^{\frac{d}{d t}}(J(x-1, m))$. Then $U=\operatorname{span}_{\mathbb{k}}\left\{\sum_{i, j} \alpha_{i j}^{(k)} e_{i j} \mid k=1, \ldots, m\right\}$.

In $\mathbb{P}\left(\bigwedge^{m} J(x-1, m)\right)$ to the subspace $U$ is associated a line spanned by $\omega_{U}=$ $\left(\sum_{i, j} \alpha_{i j}^{(1)} e_{i j}\right) \wedge \ldots \wedge\left(\sum_{i, j} \alpha_{i j}^{(m)} e_{i j}\right)$. It is easy to see that coefficients in the decomposition $\omega_{U}=\sum_{q l_{1}+\nu_{1}<\ldots<q l_{m}+\nu_{m}} p_{q l_{1}+\nu_{1}, \ldots, q l_{m}+\nu_{m}} e_{\nu_{1} l_{1}} \wedge \ldots \wedge e_{\nu_{m} l_{m}}$ satisfy the relations (ii).

Now let $\omega_{U}=\left(\sum_{i, j} \alpha_{i j}^{(1)} e_{i j}\right) \wedge \ldots \wedge\left(\sum_{i, j} \alpha_{i j}^{(m)} e_{i j}\right)=\sum_{L=\left(q l_{1}+\nu_{1}<\ldots<q l_{m}+\nu_{m}\right)} p_{L} e_{L}$ be a tensor corresponding to a subspace $U$. One can easily check that $U$ is $\frac{d}{d t}$ invariant if and only if $\omega_{U}$ is a relative $\frac{d}{d t}$-invariant. On the other hand, $\frac{d}{d t} \cdot \omega_{U}=$ $\left(\sum_{i, j} \alpha_{i j}^{(1)} \frac{d}{d t} e_{i j}\right) \wedge \ldots \wedge\left(\sum_{i, j} \alpha_{i j}^{(m)} \frac{d}{d t} e_{i j}\right)=\left(\sum_{i}\left(\alpha_{i 0}^{(1)} e_{i 0}+\alpha_{i 1}^{(1)}\left(e_{i 1}+e_{i 0}\right)+\ldots+\right.\right.$ $\left.\left.\alpha_{i, m-1}^{(1)}\left(e_{i, m-1}+e_{i, m-2}\right)\right)\right) \wedge \ldots \wedge\left(\sum_{i}\left(\alpha_{i 0}^{(m)} e_{i 0}+\alpha_{i 1}^{(m)}\left(e_{i 1}+e_{i 0}\right)+\ldots+\alpha_{i, m-1}^{(m)}\left(e_{i, m-1}+\right.\right.\right.$ $\left.\left.\left.e_{i, m-2}\right)\right)\right)=\omega_{U}+¥_{U}$. The last term denoted by $¥_{U}$ is to be investigated. Its summands are obtained when $e_{i, j-1}$ are taken instead of $e_{i j}$ in the above wedge product. Therefore, the coefficient of $e_{\nu_{1} l_{1}} \wedge \ldots \wedge e_{\nu_{m} l_{m}}$ in this term equals

$$
\sum_{\substack{\epsilon_{1}, \ldots, \epsilon_{m} \in\{0 ; 1\}^{m} \\ \epsilon_{1}^{2}+\ldots+\epsilon_{m}^{2} \neq 0}} p_{q l_{1}+\nu_{1}+q \epsilon_{1}, \ldots, q l_{m}+\nu_{m}+q \epsilon_{m}} .
$$

But let $l_{1}^{*}, \ldots, l_{m}^{*}$ be such a tuple that $p_{q r_{1}+\eta_{1}, \ldots, q r_{m}+\eta_{m}}=0$ or not defined for all vectors $\left(q r_{1}+\eta_{1}, \ldots, q r_{m}+\eta_{m}\right)$ with $r_{i}>l_{i}^{*} \forall i$, and, moreover, $p_{q r_{1}+\mu_{1}, \ldots, q r_{m}+\mu_{m}} \neq$ 0 for some $\mu_{1}, \ldots, \mu_{m}$. Then the coefficient $e_{\mu_{1} r_{1}} \wedge e_{\mu_{m} r_{m}}$ of $\frac{d}{d t} \cdot \omega_{U}$ equals $p_{q r_{1}+\mu_{1}, \ldots, q r_{m}+\mu_{m}}$, and thus if $\omega_{U}$ is a relative $\frac{d}{d t}$-invariant, we have $\frac{d}{d t} \cdot \omega_{U}=\omega_{U}$. Consequently, $U$ is $\frac{d}{d t}$-invariant if and only if $¥_{U}$ is zero. But we have already shown that, rewritten in terms of Plücker coordinates of $\omega_{U}$, this condition becomes (ii).

(c) By Proposition 5.2 it is sufficient to get a realization for $\operatorname{Gr}_{2 m}^{\frac{d}{d t}}\left(J\left(x^{2}+1,2 m\right)\right)$.

For $i=1, \ldots, q, j=0, \ldots, m-1$, set $e_{i j}=\left(0, \ldots, 0, \frac{1}{j !} t^{j} \cos t, 0, \ldots, 0\right)$ and $e_{i, m+j}=\left(0, \ldots, 0, \frac{1}{j !} t^{j} \sin t, 0, \ldots, 0\right)$, where each time the only nonzero component is the $i$-th one. Now let $U \in \operatorname{Gr}_{m}^{\frac{d}{d t}}\left(J\left(x^{2}+1, m\right)\right)$. Then

$$
U=\operatorname{span}_{\mathbb{k}}\left\{\sum_{i, j} \alpha_{i j}^{(k)} e_{i j} \mid k=1, \ldots, 2 m\right\} .
$$

In $\mathbb{P}\left(\bigwedge^{2 m} J\left(x^{2}+1, m\right)\right)$ to the subspace $U$ is associated a line spanned by $\omega_{U}=\left(\sum_{i, j} \alpha_{i j}^{(1)} e_{i j}\right) \wedge \ldots \wedge\left(\sum_{i, j} \alpha_{i j}^{(2 m)} e_{i j}\right)$. It is easy to see that coefficients in the decomposition $\omega_{U}=\sum_{q l_{1}+\nu_{1}<\ldots<q l_{2 m}+\nu_{2 m}} p_{q l_{1}+\nu_{1}, \ldots, q l_{2 m}+\nu_{2 m}} e_{\nu_{1} l_{1}} \wedge \ldots \wedge e_{\nu_{2 m} l_{2 m}}$ satisfy the relations (ii). 
Now let $\omega_{U}=\left(\sum_{i, j} \alpha_{i j}^{(1)} e_{i j}\right) \wedge \ldots \wedge\left(\sum_{i, j} \alpha_{i j}^{(2 m)} e_{i j}\right)=\sum_{L=\left(q l_{1}+\nu_{1}<\ldots<q l_{2 m}+\nu_{2 m}\right)} p_{L} e_{L}$ be a tensor corresponding to a subspace $U$. Again $U$ is $\frac{d}{d t}$-invariant if and only if $\omega_{U}$ is a relative $\frac{d}{d t}$-invariant. On the other hand, $\frac{d}{d t} \cdot \omega_{U}=\left(\sum_{i, j} \alpha_{i j}^{(1)} \frac{d}{d t} e_{i j}\right) \wedge$ $\ldots \wedge\left(\sum_{i, j} \alpha_{i j}^{(2 m)} \frac{d}{d t} e_{i j}\right)=\left(\sum_{i}\left(-\alpha_{i 0}^{(1)} e_{i m}+\alpha_{i 1}^{(1)}\left(-e_{i, m+1}+e_{i 0}\right)+\ldots+\alpha_{i m}^{(1)}\left(-e_{i, 2 m}+\right.\right.\right.$ $\left.\left.\left.e_{i, m-1}\right)\right)+\alpha_{i, m}^{(1)} e_{i 0}+\alpha_{i, m+1}^{(1)}\left(e_{i, 1}+e_{i m}\right)+\ldots+\alpha_{i, 2 m-1}^{(1)}\left(e_{i, m-1}+e_{i, 2 m-2}\right)\right) \wedge \ldots \wedge$ $\left(\sum_{i}\left(-\alpha_{i 0}^{(2 m)} e_{i m}+\alpha_{i 1}^{(2 m)}\left(-e_{i, m+1}+e_{i 0}\right)+\ldots+\alpha_{i m}^{(2 m)}\left(-e_{i, 2 m}+e_{i, m-1}\right)\right)+\alpha_{i, m}^{(2 m)} e_{i 0}+\right.$ $\left.\alpha_{i, m+1}^{(2 m)}\left(e_{i, 1}+e_{i m}\right)+\ldots+\alpha_{i, 2 m-1}^{(2 m)}\left(e_{i, m-1}+e_{i, 2 m-2}\right)\right)$. Computing the coefficient of $e_{\nu_{1} l_{1}} \wedge \ldots \wedge e_{\nu_{2 m} l_{2 m}}$ in the difference $\omega_{U}-\frac{d}{d t} \cdot \omega_{U}$, we come to the equations (ii). This completes the proof of the theorem.

Example 5.4. For $m=q=2$ these relations are very simple. It is a straightforward computation to check that in this case, for $\mu \neq \lambda$,

$$
\pi_{s}^{-1}((x-\lambda)(x-\mu)) \cong \mathbb{P}^{1} \times \mathbb{P}^{1},
$$

which is a nondegenerate quadric. When the eigenvalues coincide, the fiber is

$$
\pi_{s}^{-1}\left((x-\lambda)^{2}\right) \cong\left\{\omega=\sum_{k<l} p_{k l} e_{k} \wedge e_{l} \in \mathbb{P}\left(\bigwedge^{2} \mathbb{k}^{4}\right) \mid \begin{array}{l}
p_{14}^{2}-p_{13} p_{24}=0 \\
p_{34}=0, p_{14}=p_{23}
\end{array}\right\}
$$

i.e. a degenerate quadric. Now, if $\mathbb{k}=\mathbb{R}$ and $h$ is an irreducible quadratic polynomial, then

$$
\pi_{s}^{-1}(h) \cong\left\{\omega=\sum_{k<l} p_{k l} e_{k} \wedge e_{l} \in \mathbb{P}\left(\bigwedge^{2} \mathbb{k}^{4}\right) \mid \begin{array}{c}
p_{13}^{2}+p_{14}^{2}-p_{12} p_{34}=0 \\
p_{24}=p_{13}, p_{23}=-p_{14}
\end{array}\right\} .
$$

This real quadric is nondegenerate, but it is not isomorphic to $\mathbb{R} \mathbb{P}^{1} \times \mathbb{R} \mathbb{P}^{1}$.

Now let $Q$ be an arbitrary quiver of type $A_{n}^{(1)}$ (we use the notation from the previous section for its vertices and arrows). As was shown before, the fiber of $\mathcal{M}^{s}(Q, \alpha, \zeta)$ over a point $\bar{\chi}=\left(\chi_{1}, \ldots, \chi_{n}\right)$ of the standard categorical quotient is isomorphic to $\operatorname{Gr}_{\underline{r}_{0}}^{\mathbb{k} Q}\left(J\left(h_{0}, \underline{r}_{0}\right)\right) \times \prod_{j=1}^{N} \operatorname{Gr}_{r_{1 j} \operatorname{deg}\left(h_{j}\right)}^{\mathbb{k} A_{0}^{(1)}}\left(J\left(h_{j}, r_{1 j} \operatorname{deg}\left(h_{j}\right)\right)\right)$, where $\underline{r}_{0}=$ $\left(r_{10}, \ldots, r_{n 0}\right)$, where the notation is as in Theorem 4.7 . For $\mathbb{k}=\mathbb{R}$ of $\mathbb{C}$ we have already described all the factors of the product except the first one. It is thus left to give a realization of the null fiber $\pi_{s}^{-1}(0)$.

Fix a dimension vector $\alpha$. Recall that

$$
\operatorname{Gr}_{\alpha}^{\mathbb{k} Q}\left(J\left(h_{0}, \alpha\right)\right)=\left\{\left(N_{i} \subseteq \mathcal{A}[t] \otimes \bigoplus_{j=1}^{m} V_{j}\right)_{i=1}^{n} \begin{array}{c}
\frac{d}{d t}\left(N_{i}\right) \subseteq N_{i} \\
a_{i}\left(N_{i}\right) \subseteq N_{i+1} \\
\left.\left(\frac{d}{d t}\right)^{\max _{i} \alpha_{i}}\right|_{N_{i}} \equiv 0
\end{array}\right\},
$$

where $a_{i}: \mathcal{A}[t] \otimes \bigoplus_{j=1}^{m} V_{j} \rightarrow \mathcal{A}[t] \otimes \bigoplus_{j=1}^{m} V_{j}$ acts as $\frac{d}{d t}$ on $\mathcal{A}[t] \otimes V_{i}$ and trivially on all other components.

It is convenient to fix the following basis in all $J\left(h_{0}, \beta\right)_{i}: e_{q r s}^{(i)}=\left(0, \ldots, t^{s}, \ldots, 0\right) \in$ $\mathcal{A}[t] \otimes V_{q}$, where the only nonzero component is the $r$-th one. Furthermore, in $\operatorname{Gr}_{\alpha_{i}}\left(J\left(h_{0}, \alpha\right)_{i}\right)$ we will be considering Plucker's coordinates corresponding to this basis. They will be denoted by $p_{k_{1}, \ldots, k_{\alpha_{i}}}^{(i)}$, where $k_{h}$ are in fact triples of indices $\left(q_{h}, r_{h}, s_{h}\right)$. 
Theorem 5.5. Let $Q$ be a cyclic quiver of type $A_{n}^{(1)}$. The Grassmannian $\operatorname{Gr}_{\alpha}^{\mathbb{k} Q}\left(J\left(h_{0}, \alpha\right)\right)$ is isomorphic to the subvariety of $\prod_{i=1}^{n} \mathbb{P}\left(\bigwedge^{\alpha_{i}}\left(J\left(h_{0}, \alpha\right)\right)_{i}\right)$ given by:

(a) The equations from Theorem $5.3(\mathrm{~b})$ for each component $\mathbb{P}\left(\bigwedge^{\alpha_{i}}\left(J\left(h_{0}, \alpha\right)\right)_{i}\right)$;

(b) $\sum_{l=0}^{\alpha_{i+1}}(-1)^{l} p_{j_{1}^{\prime} \ldots j_{\alpha_{i}-1}^{\prime} k_{l}^{\prime}}^{(i)} p_{k_{0} \ldots \widehat{k}_{l} \ldots k_{\alpha_{i+1}}}^{(i+1)}=0$, for all tuples $j_{1}, \ldots, j_{\alpha_{i}-1}$ (the last component in these triples has to be less than $\left.\alpha_{i}\right)$ and $k_{1}, \ldots, k_{\alpha_{i+1}}$, where $k_{l}^{\prime}=(q, r, s+1)$ if $k_{l}=(q, r, s)$ with $q=i$, and $k_{l}^{\prime}=k_{l}$ otherwise.

Proof. At first, all $M_{i}$ are $\frac{d}{d t}$-invariant, so the statement of Theorem 5.3(b) holds. In addition, there is a condition $a_{i}\left(M_{i}\right) \subseteq M_{i+1}$. Let $\omega_{M_{i}} \in P\left(\bigwedge^{\alpha_{i}}\left(J\left(h_{0}, \alpha\right)\right)_{i}\right)$ and $\omega_{M_{i+1}} \in P\left(\bigwedge^{\alpha_{i+1}}\left(J\left(h_{0}, \alpha\right)\right)_{i+1}\right)$ be the tensors corresponding to $M_{i}$ and $M_{j}$; also let $\omega_{M_{i}}^{\prime}=a_{i} \cdot \omega_{M_{i}}$. Consider the basis $\xi_{q r s}^{(i+1)}$ in $\bigwedge^{\alpha_{i}}\left(J\left(h_{0}, \alpha\right)\right)_{i}$ that is adjoined to $e_{q, r, s}^{(i)}$. Then $a_{i}\left(M_{i}\right) \subseteq M_{i+1}$ if and only if for each tuple $j_{1}, \ldots, j_{\alpha_{i}-1}$ we have $\omega_{M_{i}}^{\prime}\left(\xi_{j_{1}}^{(i+1)}, \ldots, \xi_{j_{\alpha_{i}-1}}^{(i+1)}, \cdot\right) \wedge \omega_{M_{i+1}}=0$. But, on the other hand, $\left\langle a_{i}\left(e_{k}^{(i)}\right), \xi_{j}^{(i+1)}\right\rangle \neq 0$ if and only if $k=j^{\prime}$ (the meaning of prime was defined in the statement of the theorem), so $\omega_{M_{i}}^{\prime}\left(\xi_{j_{1}}^{(i+1)}, \ldots, \xi_{j_{\alpha_{i}-1}}^{(i+1)}, \cdot\right)=\sum_{l} p_{j_{1}^{\prime} \ldots j_{\alpha_{i}-1}^{\prime} l^{\prime}}^{(i)} e_{l}^{(i+1)}$. Consequently, $\omega_{M_{i}}^{\prime}\left(\xi_{j_{1}}^{(i+1)}, \ldots, \xi_{j_{\alpha_{i}-1}}^{(i+1)}, \cdot\right) \wedge \omega_{M_{i+1}}=\left(\sum_{l} p_{j_{1}^{\prime} \ldots j_{\alpha_{i}-1}^{\prime} l^{\prime}}^{(i)} e_{l}^{(i+1)}\right) \wedge\left(\sum p_{h_{1} \ldots h_{\alpha_{i+1}}}^{(i+1)} e_{h_{1}}^{(i+1)} \wedge\right.$ $\left.\ldots \wedge e_{h_{\alpha_{i+1}}}^{(i+1)}\right)$. Having opened the brackets, we obtain that the coefficient of $e_{k_{0}}^{(i+1)} \wedge$ $e_{k_{1}}^{(i+1)} \ldots e_{k_{\alpha_{i+1}}}^{(i+1)}$ equals $\pm \sum_{l=0}^{\alpha_{i+1}}(-1)^{l} p_{j_{1}^{\prime} \ldots j_{\alpha_{i}-1}^{\prime} k_{l}^{\prime}}^{(i)} p_{k_{0} \ldots \widehat{k}_{l} \ldots k_{\alpha_{i+1}}}^{(i+1)}$. But as was mentioned before, the inclusion $a_{i}\left(M_{i}\right) \subseteq M_{i+1}$ is equivalent to the fact that all $\omega_{M_{i}}^{\prime}\left(\xi_{j_{1}}^{(i+1)}, \ldots, \xi_{j_{\alpha_{i}-1}}^{(i+1)}, \cdot\right) \wedge \omega_{M_{i+1}}$ are zero, which means that their coefficients are zero. Thus we come to (b).

\section{Quivers WITH SUCCESSIVE CYClES}

A quiver $Q$ will be called a quiver with successive cycles if whenever two oriented cycles in $Q$ have a common vertex, they are both powers of a certain cycle. It is easy to see that all such quivers may be constructed through the following procedure (that justifies our choice of terminology). Take a quiver without oriented cycles and replace some of its vertices by oriented cycles so that the arrows that used to start from the replaced vertex may now start from any chosen vertex of the pasted cycle. Here is an example of a quiver obtained through such a transformation:

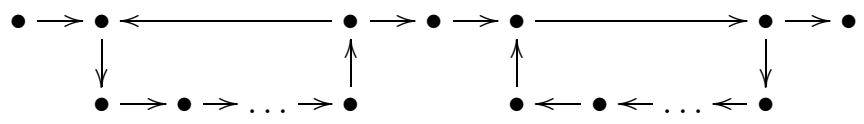

Using our description of the quotient for the case of one oriented cycle, we can generalize the technique we possess to such quivers. As before, we denote by $\pi$ the natural map $\operatorname{Rep}^{s}(Q, \alpha, \zeta) \rightarrow \operatorname{Spec} \mathbb{k}[\operatorname{Rep}(Q, \alpha, \zeta)]^{G L(\alpha)}$.

Theorem 6.1. Let $Q$ be a quiver with successive cycles. Also let $\alpha$ and $\zeta$ be two dimension vectors and $y$ be a point in $\operatorname{Spec} \mathbb{k}[\operatorname{Rep}(Q, \alpha, \zeta)]^{G L(\alpha)}$. There exists a quiver $Q^{\wedge}$, a dimension vector $\widetilde{\alpha} \in\left(\mathbb{Z}_{\geqslant 0}\right)^{Q_{0}^{\wedge}}$, and a finite dimensional representation $W$ of $Q^{\uparrow}$ such that $\pi^{-1}(y) / / G L(\alpha) \cong \operatorname{Gr}_{\widetilde{\alpha}}^{\mathbb{k} Q^{\star}}$ (W $W^{\star}$. 
Proof. We begin the proof by giving a construction of a module $J$ such that points in $\operatorname{Gr}_{\alpha}^{\mathbb{k} Q}(N)$ are in one-to-one correspondence with points in the orbit space $\mathcal{M}^{s}(Q, \alpha, \zeta)$.

Let $\widehat{Q}$ be the quiver without oriented cycles, from which $Q$ may be obtained through the above procedure, and $\Xi_{i}$ be the set of all paths in $\widehat{Q}$ starting at $i$. For the vertices of the oriented cycle that we place instead of the $i$-th vertex of $\widehat{Q}$, we set the following notation:

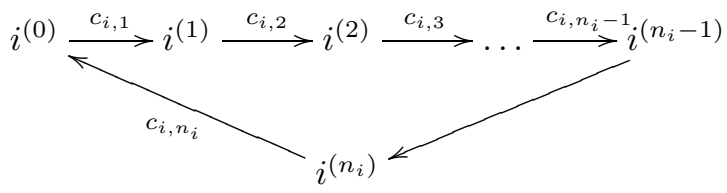

Also let $\tau_{i, j}$ be the cycle of minimal nonzero length starting at $i^{(j)}$, if there is any, or $e_{i}=e_{i(0)}$ otherwise.

As before, we consider the $\mathbb{k} Q$-module $J$ with $J_{i}=\prod_{\sigma: i \rightsquigarrow j} V_{j}^{\sigma}$. Every path $\sigma$ in $Q$ is of the form $\sigma=B_{l} \tau_{i_{l}, j_{l}}^{k_{l}} a_{l} \ldots B_{2} \tau_{i_{2}, j_{2}}^{k_{2}} a_{2} B_{1} \tau_{i_{1}, j_{1}}^{k_{1}} a_{1} B_{0} \tau_{i_{0}, j_{0}}^{k_{0}}$, where $l=l(\sigma)$ is the length of $\sigma, a_{j}$ are arrrows of $\widehat{Q}$, and $B_{t}$ are segments of $\tau_{i_{t}, j_{t}}$, that are not oriented cycles. Let $a_{0}=i$ and $u_{t}=h a_{t}$. We then have that, for $r=0, \ldots, n_{u_{l}}$,

$$
J_{i(r)} \cong \prod_{\Xi_{i} \ni \rho=a_{l} \ldots a_{2} a_{1}} \bigoplus_{t=0}^{\max \left(n_{u_{l}}-1,0\right)} K_{l}\left[x_{\rho, l}\right] \otimes \ldots \otimes K_{0}\left[x_{\rho, 0}\right] \otimes V_{u_{l}^{(t)}}
$$

where

$$
K_{q}[x]:=\left\{\begin{array}{l}
\mathbb{k}[[x]], \text { if } l\left(\tau_{i_{q}, j_{q}}\right)>0 \\
\mathbb{k}, \text { otherwise. }
\end{array}\right.
$$

Here we use $\mathbb{k}[[x]]$ instead of $c_{\infty}$ to be able to distinguish components corresponding to different cycles without using additional multiple indices. All the operations on power series are interpreted as direct translations of those on sequences. For instance, the left shift corresponds to the left shift and not to formal differentiation.

Let $c_{1}=c_{u_{l}, 1}$ be the arrow belonging to $\tau_{i_{l}, j_{l}}$ that starts at $u_{l}$. For a pair $(M, f) \in \operatorname{Rep}(Q, \alpha, \zeta)$ the map $\Phi_{(M, f)}: M \rightarrow J ! !$ acts as

(9) $\varphi_{i(r)}=\bigoplus_{\Xi_{i} \ni \rho=a_{l} \ldots a_{2} a_{1}} \bigoplus_{t=0}^{\max \left(n_{u_{l}}-1,0\right)} f_{i_{l}^{t+1}} c_{t} \ldots c_{1} \mathfrak{E} \mathfrak{x p}\left(\tau_{i_{l}, j_{l}} x_{\rho, l}\right) \ldots a_{1} B_{0} \mathfrak{E} \mathfrak{k} \mathfrak{p}\left(\tau_{i_{0}, r} x_{\rho, 0}\right)$,

where

$$
\mathfrak{E x p}\left(\tau_{i, j} y\right):=\left\{\begin{array}{l}
\mathfrak{e x p}\left(\tau_{i, j} y\right), \text { if } l\left(\tau_{i, j}\right)>0, \\
\mathrm{id}, \text { otherwise. }
\end{array}\right.
$$

Combining the proofs of Proposition 3.3 and Lemma 4.1 we see that the thus defined $\Phi_{(M, f)}$ enjoys its usual properties, i.e. that the statement of Lemma 4.1 holds in this situation.

Proposition 6.2. The map $\Phi: \operatorname{Rep}^{s}(Q, \alpha, \zeta) \rightarrow \operatorname{IHom}_{\alpha}^{\mathbb{k} Q}(J),(M, f) \mapsto \Phi_{(M, f)}$ is a bijection.

Proof. Let $U \subseteq J$ be a subrepresentation with dimension vector $\alpha$. We need a pair $(M, f)$ such that $\operatorname{Im}\left(\Phi_{(M, f)}\right)=U$. Take $M=U$. The maps $f_{i^{(j)}}$ are then 
reconstructed as compositions of the projections $U_{i^{(j)}} \rightarrow \mathcal{K}_{0}\left[x_{e_{i}, 0}\right] \otimes V_{i^{(j)}}$ with an evaluation at 0 . It is now only left to show that $\Phi_{(U, f)}$ is the natural inclusion $U \hookrightarrow J$.

The proof is by "downward induction over $\widehat{Q}$ ". If $i \in \widehat{Q}$ is a sink, we apply Proposition 4.2. For an arbitrary vertex $i$, fix a basis $G_{1}, \ldots, G_{d_{j}}$ in each $U_{i^{(j)}}$ and observe that, as in (5),

$$
\left(G_{1}\left(x_{e_{i}, 0}\right), \ldots, G_{d_{j}}\left(x_{e_{i}, 0}\right)\right)=\left(G_{1}(0), \ldots, G_{d_{j}}(0)\right) \mathfrak{e x p}\left(\tau_{i, j} x_{e_{i}, 0}\right),
$$

where $G_{p}\left(x_{e_{i}}, 0\right)$ are in fact functions in $x_{\rho, t}$, for $\rho \neq e_{i}$. We need to prove that the right hand side of this equality coincides with (9). Recall that $\bigoplus_{a: i \rightarrow p} a B_{0, a}$, where $B_{0, a}$ is the shortest segment of $\tau_{i, j}$ linking $i^{j}$ with $t a$, acts as an evaluation at $x_{e_{i}, 0}=0$. So, $\left(G_{1}(0), \ldots G_{d_{j}}(0)\right)$ consists of horizontal blocks representing the bases of $a B_{0, a}\left(U_{i^{j}}\right)$, where $a$ are arrows starting at vertices of $\tau_{i, j}$. By the induction hypothesis all these blocks are of the form (9). Thus the claim follows.

This, however, characterizes neither $\operatorname{Gr}_{\alpha}^{\mathbb{k} Q}(J)$ nor the orbit space. As in Section 4, we restrict our attention to the fibers of $\pi$.

Recall that the algebra $\mathbb{k}[\operatorname{Rep}(Q, \alpha, \zeta)]$ is generated by coefficients of characteristic polynomials of all oriented cycles in $Q$. So we can consider $y$ as a tuple $\left\{\widehat{\chi}_{i, j} \mid i \in \widehat{Q}_{0}, j=0, \ldots, n_{i}\right\}$, where

$$
\widehat{\chi}_{i, j}:=\left\{\begin{array}{l}
\text { the characteristic polynomial of } \tau_{i, j}, \text { if } l(\tau(i, j)>0, \\
0, \text { otherwise. }
\end{array}\right.
$$

We are now ready to prove the theorem. Introduce the module $W$ with

$$
W_{i(r)}:=\bigoplus_{\Xi_{i} \ni \rho=a_{l} \ldots a_{2} a_{1}}\left(\bigotimes_{t=0}^{l} \mathcal{K}_{t}\left[x_{\rho, t}\right] \otimes\left(\bigoplus_{t=0}^{\max \left(n_{u_{l}}-1,0\right)} V_{u_{l}^{(t)}}\right)\right)_{\substack{\widehat{\chi}_{i t}, j_{t}\left(\delta_{x_{\rho, t}}\right), t=0, \ldots, l}},
$$

where $j_{0}=r$. It is clearly finite dimensional. We claim that for a stable pair $(M, f) \in \pi^{-1}(y)$ the image of $\varphi_{(M, f)}$ lies in $W$.

We prove this by "downward induction over $\widehat{Q}$ ". Let $i$ be a $\operatorname{sink}$ in $\widehat{Q}$. If this vertex is replaced by an oriented cycle, we may use the results of Section 4; otherwise the claim is trivial.

Now, consider an arbitrary vertex $i$. If it does not belong to an oriented cycle, recall that by Proposition 3.8 we have, for every submodule $U$ of $J, U_{i^{(0)}} \subseteq V_{i^{(0)}} \oplus$ $\bigoplus_{a: i^{(0)} \rightarrow j^{(r)}} U_{j(r)} \subseteq V_{i^{(0)}} \oplus \bigoplus_{a: i^{(0)} \rightarrow j^{(r)}} W_{j^{(r)}}$, but by definition of $\widehat{\chi}_{i, 0}$ this clearly lies in $W_{i(0)}$. Otherwise, we use (9) with $\mathfrak{e x p}$ the usual matrix exponents to understand that all $\widehat{\chi}_{i_{t}, j_{t}}\left(\delta_{x_{\rho, t}}\right)$ annulate $W_{i(r)}$, for $r=0, \ldots, n_{i}$.

Although $\Phi:(M, f) \mapsto \Phi^{(M, f)}$ induces an inclusion $\pi_{G L(\alpha)}^{-1}(y) \cap \operatorname{Rep}^{s}(Q, \alpha, \zeta) \hookrightarrow$ $\operatorname{IHom}_{\alpha}^{\mathbb{k} Q}(W)$, in general it is not surjective. For example, let $Q$ be a Jordan quiver consisting of a single loop $a$. Take $\alpha=\zeta=2$ and $\chi_{a}(x)=(x-1)(x-2)$. Then the corresponding $W$ contains a subrepresentation $U$ spanned by $\left(\begin{array}{c}e^{t} \\ 0\end{array}\right)$ and $\left(\begin{array}{c}0 \\ e^{t}\end{array}\right)$, which is not in $\pi_{G L(2)}^{-1}\left(\chi_{a}\right)$. Hence we need to refine our construction.

Let $h_{0}^{(i)}=x_{e_{i}, 0}, h_{1}^{(i)}, \ldots, h_{N_{i}}^{(i)}$ be all different irreducible factors of $\widehat{\chi}_{i, j}$, for $j=$ $1, \ldots, n_{i}$. Also let $r_{l}^{(i, j)}$ be the multiplicity of $h_{l}^{(i)}$ as a factor of $\widehat{\chi}_{i, j}$ and $\underline{r}_{l}^{(i)}=$ $\left(r_{l}^{(i, 0)} \operatorname{deg}\left(h_{e}^{(i)}\right), \ldots, r_{l}^{\left(i, n_{i}\right)} \operatorname{deg}\left(h_{e}^{(i)}\right)\right)$. If $\widehat{\chi}_{i, j}$ is a zero polynomial, we set $N_{i}=1$, 
$h_{1}^{(i)}=0$, and $r_{1}^{(i, 0)}=\alpha_{i^{(j)}}$. For $j=0, \ldots, n_{i}$, define $J\left(h_{l}^{(i)}, \underline{r}_{l}^{(i)}\right)_{i^{(j)}}$ as the subspace of $W_{i^{(j)}}$ generated by all solutions of the equation $\left(h_{l}^{(i)}\left(\delta_{x_{e_{i}, 0}}\right)\right)^{\max _{q} r_{l}^{(i, q)}} \bar{g}=0$. Note that usually these subspaces do not form a subrepresentation, as they used to in Section 4. Since $W_{i^{(j)}}=\bigoplus_{l=1}^{N_{i}}\left(W_{i^{(j)}} \cap J\left(h_{l}^{(i)}, \underline{r}_{l}^{(i)}\right)_{i^{(j)}}\right)$, there are natural projections $\mathbf{p}_{l}^{(i, j)}: W_{i^{(j)}} \rightarrow W_{i^{(j)}} \cap J\left(h_{l}^{(i)}, \underline{r}_{l}^{(i)}\right)_{i^{(j)}}$ along $\bigoplus_{l^{\prime} \neq l}^{N_{i}}\left(W_{i^{(j)}} \cap J\left(h_{l^{\prime}}^{(i)}, \underline{r}_{l^{\prime}}^{(i)}\right)_{i^{(j)}}\right)$. The same arguments as we used to prove Lemma 4.5 show that a stable pair $(M, f)$ lies in $\pi_{G L(\alpha)}^{-1}(y)$ if and only if the dimensions of $\mathbf{p}_{l}^{(i, j)}\left(\Phi_{(M, f)}(M)_{i^{(j)}}\right)$ equal $r_{l}^{(i, j)} \operatorname{deg}\left(h_{e}^{(i)}\right)$, for all $i, j$ and $l$. Hence, $\Phi$, induces an inclusion of $\pi_{G L(\alpha)}^{-1}(y) \cap$ $\operatorname{Rep}^{s}(Q, \alpha, \zeta)$ into the subvariety $\widetilde{\mathrm{IH}}(W)$ in

$$
\prod_{\substack{i^{(j)} \in \mathbb{k} Q_{0}, l=1, \ldots, N_{i}}} \operatorname{IHom}_{r_{l}^{(i, j)}} \operatorname{deg}\left(h_{e}^{(i)}\right)\left(W_{i^{(j)}} \cap J\left(h_{l}^{(i)}, \underline{r}_{l}^{(i)}\right)_{i^{(j)}}\right),
$$

consisting of those tuples of injections whose images give a $Q$-subrepresentation of $W$. Using Lemma 3.6 one may deduce that this inclusion is in fact a $G L(\alpha)$ invariant isomorphism of algebraic varieties. Therefore, $\pi^{-1}(y) / / G L(\alpha) \cong \widetilde{\mathrm{IH}}(W) / /$ $G L(\alpha)$. However, the latter does not yet look like a Grassmannian of submodules.

We now construct a quiver $Q^{\boldsymbol{\omega}}$, setting

$$
Q_{0}^{\boldsymbol{\phi}}=\left\{i^{(j, l)} \mid i^{(j)} \in Q_{0}, l=1, \ldots, N_{i}\right\}
$$

and

$$
\begin{aligned}
Q_{1}^{\mathbf{\phi}}= & \left\{c_{i, j, l}: i^{(j, l)} \rightarrow i^{(j+1, l)} \mid j=0, \ldots, n_{i} ; l=1, \ldots, N\right\} \\
& \cup\left\{a^{\left(l_{1}, l_{2}\right)}: i_{1}^{\left(j_{1}, l_{1}\right)} \rightarrow i_{2}^{\left(j_{2}, l_{2}\right)} \mid a: i_{1}^{j_{1}} \rightarrow i_{2}^{j_{2}}\right\} .
\end{aligned}
$$

Observe that $W$ may be considered as a representation $W^{\boldsymbol{\omega}}$ of $Q^{\boldsymbol{\omega}}$ with $W_{i(j, l)}^{\boldsymbol{\phi}}=$ $W_{i^{(j)}} \cap J\left(h_{l}^{(i)}, \underline{r}_{l}^{(i)}\right)_{i^{(j)}}$. The dimension vector of $W^{\boldsymbol{\omega}}$ is $\widetilde{\alpha}$ with $\widetilde{\alpha}_{i^{(j, l)}}=r_{l}^{(i, j)} \operatorname{deg}\left(h_{e}^{(i)}\right)$. It is now straightforward to check that $\widetilde{\mathrm{IH}}(W) / / G L(\alpha)$ is isomorphic to the Grassmannian $\operatorname{Gr}_{\widetilde{\alpha}}^{\mathbb{k} Q^{\star}}\left(W^{\star}\right)$. This finishes the proof of Theorem 6.1.

Remark 6.3. For $Q=A_{n-1}^{(1)}$ the quiver $Q^{\infty}$ is nonconnected. In fact, it is a union of $(N+1)$ copies of $Q$, where $N$ is as in Theorem 4.7. So, a $\mathbb{k} Q$-module is an $N$-tuple of representations of $Q$. In particular, $W^{\boldsymbol{\omega}}$ splits as $\bigoplus_{j} J\left(h_{j}, \underline{r}_{j}\right)$. So, $\operatorname{Gr}_{\widetilde{\alpha}}^{\mathbb{k} Q}\left(W^{\star}\right) \cong \prod_{j} \operatorname{Gr}_{\underline{r}_{j}}^{\mathbb{k} Q}\left(J\left(h_{j}, \underline{r}_{j}\right)\right)$.

Example 6.4. Let $\mathbb{k}=\mathbb{C}$ and $\widehat{Q}=1 \underset{a_{1}^{\prime \prime}}{\stackrel{a_{1}^{\prime}}{\longrightarrow}} 2 \stackrel{a_{2}}{\longrightarrow} 3 \stackrel{a_{3}}{\longrightarrow} 4 \stackrel{a_{4}}{\longrightarrow} 5$. Replace its second and fourth vertices by loops $b$ and $c$ respectively, and denote the quiver obtained by $Q$.

Let $\left(\chi_{b}, \chi_{c}\right) \in \mathbb{k}[x]_{\alpha_{2}} \oplus \mathbb{k}[x]_{\alpha_{4}}$. Also let $\chi_{b}=\prod_{i=0}^{N_{b}}\left(x-\lambda_{i}^{(b)}\right)^{r_{i}^{(b)}}$ and $\chi_{b}=$ $\prod_{i=0}^{N_{b}}\left(x-\lambda_{i}^{(c)}\right)^{r_{i}^{(c)}}$, where $\lambda_{0}^{(b)}=\lambda_{0}^{(c)}=0$ and $\lambda_{i}^{(b)} \neq \lambda_{j}^{(b)}, \lambda_{i}^{(c)} \neq \lambda_{j}^{(c)}$, for $i \neq j$. Then the quiver $Q^{\boldsymbol{\omega}}$ has vertices $Q_{0}^{\boldsymbol{\phi}}=\left\{1,2_{0}, 2_{1}, \ldots, 2_{N_{b}}, 3,4_{0}, 4_{1}, \ldots, 4_{N_{c}}, 5\right\}$ and 
arrows $a_{1 j}^{\prime}, a_{1 j}^{\prime}{ }^{\prime}: 1 \rightarrow 2_{j}, b_{j}: 2_{j} \rightarrow 2_{j}, a_{2 j}: 2_{j} \rightarrow 3, a_{3 l}: 3 \rightarrow 4_{l}, c_{l}: 4_{l} \rightarrow 4_{l}$, and $a_{4 l}: 4_{l} \rightarrow 5$, for $j=0,1, \ldots, N_{b}, l=0,1, \ldots, N_{c}$. Thus, $Q^{\mathbf{s}}$ is

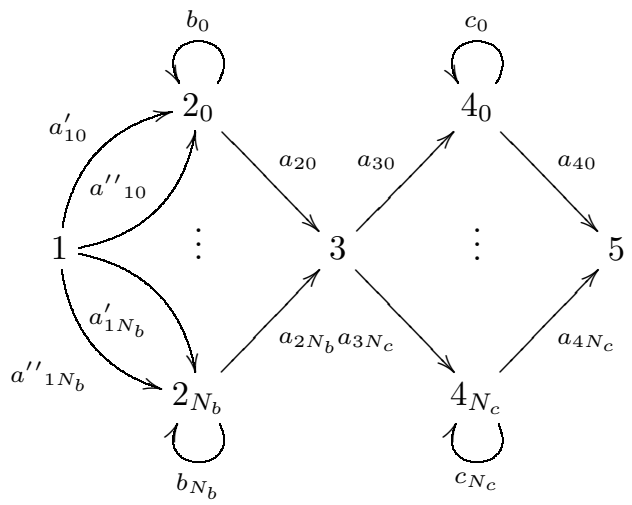

and $\widetilde{\alpha}$ is the dimension vector with $\widetilde{\alpha}_{i}=\alpha_{i}$, for $i \in\{1,3,5\}, \widetilde{\alpha}_{2_{j}}=r_{j}^{(b)}$, and $\widetilde{\alpha}_{4_{l}}=r_{l}^{(c)}$.

On the other side, the semisimple representation lying in the orbit closure of a representation with the above characteristic polynomials $\left(\chi_{b}, \chi_{c}\right)$ is

$$
T:=S_{1} \oplus S_{3} \oplus S_{5} \oplus r_{0}^{(b)} S_{2} \oplus r_{0}^{(c)} S_{4} \oplus \bigoplus_{i=1}^{N_{b}} r_{i}^{(b)} E_{\lambda_{i}^{(b)}}^{(b)} \oplus \bigoplus_{i=1}^{N_{c}} r_{i}^{(c)} E_{\lambda_{i}^{(c)}}^{(c)},
$$

where $E_{\lambda}^{(b)}$ and $E_{\lambda}^{(c)}$ are simple representations $E_{\lambda}$ of the Jordan quiver inserted at the second and the fourth vertices respectively. Then the new quiver $Q_{T}$ and the dimension vector $\alpha_{T}$ from [6, Theorem 4.1] are as follows. The vertices of $Q_{T}$ are indexed by pairwise nonisomorphic simple summands in $T$, i.e. $\left(Q_{T}\right)_{0}=$ $\left\{1,3,5,2_{0}, 2_{1}, \ldots, 2_{N_{b}}, 4_{0}, 4_{1}, \ldots, 4_{N_{c}}\right\}$. Moreover, vertices $i$ and $j$ corresponding to simple modules $S$ and $S^{\prime}$ in $Q_{T}$ are joined by $\delta_{i j}-\left\langle\underline{\operatorname{dim}} S, \underline{\operatorname{dim}} S^{\prime}\right\rangle_{Q}$ arrows $i \rightarrow j$, where $\langle\cdot, \cdot\rangle_{Q}$ is the Euler form of $Q$. Straightforward computation now shows that $Q_{T}$ coincides with $Q^{\star}$. Components of the dimension vector $\alpha_{T}$ are by definition multiplicities of simple summands of $T$, so it coincides with $\widetilde{\alpha}$. Moreover, the same technique as we used in the proof of Proposition 5.1 allows us to show that if $Q^{\star}$ is fixed, the Grassamnnian $\operatorname{Gr}_{\widetilde{\alpha}}^{\mathbb{k} Q}\left(W^{\star}\right)$ is up to isomorphism independent

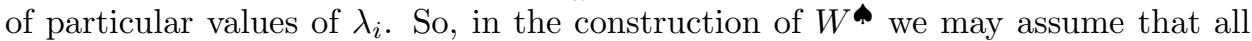
$\lambda_{i}=0$. We thus obtain the first statement of [6, Theorem 4.1] for the quiver $Q$.

One may check that this is the general rule over $\mathbb{C}$. For any quiver $Q$ with successive cycles we have $Q^{\boldsymbol{}}=Q_{T}, \widetilde{\alpha}=\alpha_{T}$ and $W^{\boldsymbol{\omega}}$ is the module $J$ from Theorem 3.5 for the algebra $\mathbb{k} Q /(\mathbb{k} Q)^{\left(\max _{i} \alpha_{i}\right)}$ and a special choice of framing $\widetilde{\zeta}$.

\section{REFERENCES}

1. M. Auslander, I. Reiten, S. O. Smalø, Representation Theory of Artin Algebras, Cambridge Studies in Advanced Math., 36, Cambridge University Press, 1995. MR1314422 (96c:16015)

2. K. Bongartz, A geometric version of the Morita equivalence, J. Algebra, 139, 1991, pp. 159171. MR:1106345 (92f:16008)

3. P. Caldero, M. Reineke, On the quiver Grassmannian in the acyclic case, J. Pure Appl. Algebra, 212, 2008, no. 11, pp. 2369-2380. MR2440252(2009f:14102)

4. S. Donkin, Polynomial invariants of representations of quivers, Comment. Math. Helv., 69, 1994, no. 1, pp. 137-141. MR 1259609 (95a:16016) 
5. Yu. Drozd, V. V. Kirichenko, Finite-dimensional algebras (with an appendix by Vlastimil Dlab), Springer-Verlag, Berlin, 1994, 249 pp. MR.1284468 (95i:16001)

6. J. Engel, M. Reineke, Smooth models of quiver modui, Math. Z., 262, 2009, pp. 817-848. MR2511752(2010h:16033)

7. B. Huisgen-Zimmermann, Classifying representations by way of Grassmannians, Trans. Amer. Math. Soc., 359, 2007, pp. 2687-2719. MR2286052 (2007k:16024)

8. B. Huisgen-Zimmermann, A hierarchy of parametrizing varieties for representations, Contemporary Mathematics, 480, 2009, pp. 207-239. MR2508153(2010h:16028)

9. A. D. King, Moduli of representations of finite-dimensional algebras, Quart. J. Math. Oxford Ser. (2), 45(180), 1994, pp. 515-530. MR1315461 (96a:16009)

10. H. Kraft, Geometrische Methoden in der Invariantentheorie, Aspekte der Mathematik vol. D1, Vieweg Verlag 1985. MR768181 (86j:14006)

11. L. Le Bruin, C. Procesi, Semisimple representations of quivers, Trans. Amer. Math. Soc. 317, 1990, pp. 585-598. MR958897 (90e:16048)

12. H. Nakajima, Varieties associated with quivers, in: Representation Theory of Algebras and Related Topics, Mexico City, 1994, in: CMS Conf. Proc., vol. 19, Amer. Math. Soc., Providence, RI, 1996, pp. 139-157. MR1388562 (97m:16022)

13. M. Reineke, Framed quiver moduli, cohomology, and quantum groups, J. Algebra, 320, 2008, no. 1, pp. 94-115. MR2417980 (2009d:16021)

14. A. Rudakov, Stability for an abelian category, J. Algebra, 197, 1997, no. 1, pp. 231-245. MR 1480783 (98j:14011)

15. A.N. Zubkov, The Razmyslov-Procesi theorem for quiver representations, Fundam. Prikl. Mat. (Russian), 7, 2001, no. 2, pp. 387-427. MR1866464(2002i:16017)

Department of Higher Algebra, Main Building, Moscow State University, GSP-1, 1 Leninskiye Gory, Moscow, 119991 Russia

E-mail address: glwrath@yandex.ru 